\title{
Search for gravitational waves associated with the gamma ray burst GRB030329 using the LIGO detectors
}

B. Abbott,${ }^{11}$ R. Abbott, ${ }^{14}$ R. Adhikari, ${ }^{12}$ A. Ageev, ${ }^{19,26}$ B. Allen, ${ }^{38}$ R. Amin,${ }^{33}$ S. B. Anderson, ${ }^{11}$ W. G. Anderson, ${ }^{28}$ M. Araya ${ }^{11}$ H. Armandula, ${ }^{11}$ M. Ashley,${ }^{27}$ F. Asiri, ${ }^{11, a}$ P. Aufmuth, ${ }^{30}$ C. Aulbert, ${ }^{1}$ S. Babak, ${ }^{7}$ R. Balasubramanian, ${ }^{7}$ S. Ballmer, ${ }^{12}$ B. C. Barish, ${ }^{11}$ C. Barker, ${ }^{13}$ D. Barker ${ }^{13}$ M. Barnes, ${ }^{11, b}$ B. Barr,${ }^{34}$ M. A. Barton, ${ }^{11}$ K. Bayer, ${ }^{12}$ R. Beausoleil, ${ }^{25, \mathrm{c}}$ K. Belczynski, ${ }^{22}$ R. Bennett, ${ }^{34, \mathrm{~d}}$ S. J. Berukoff, ${ }^{1, \mathrm{e}} \mathrm{J}$. Betzwieser, ${ }^{12}$ B. Bhawal,${ }^{11}$ I. A. Bilenko, ${ }^{19}$ G. Billingsley, ${ }^{11}$ E. Black, ${ }^{11}$ K. Blackburn, ${ }^{11}$ L. Blackburn, ${ }^{12}$ B. Bland,${ }^{13}$ B. Bochner, ${ }^{12, f}$ L. Bogue,${ }^{11}$ R. Bork, ${ }^{11}$ S. Bose,${ }^{39}$ P. R. Brady, ${ }^{38}$ V. B. Braginsky, ${ }^{19}$ J.E. Brau, ${ }^{36}$ D. A. Brown, ${ }^{38}$ A. Bullington, ${ }^{25}$ A. Bunkowski, ${ }^{2,30}$ A. Buonanno, ${ }^{6, g}$ R. Burgess, ${ }^{12}$ D. Busby, ${ }^{11}$ W.E. Butler,${ }^{37}$ R. L. Byer, ${ }^{25}$ L. Cadonati, ${ }^{12}$ G. Cagnoli, ${ }^{34}$ J. B. Camp, ${ }^{20}$ J. K. Cannizzo, ${ }^{20}$ C. A. Cantley, ${ }^{34}$ L. Cardenas, ${ }^{11}$ K. Carter, ${ }^{14}$ M. M. Casey ${ }^{34}$ J. Castiglione, ${ }^{33}$ A. Chandler, ${ }^{11}$ J. Chapsky, ${ }^{11, b}$ P. Charlton, ${ }^{11, \mathrm{~h}}$ S. Chatterji, ${ }^{12}$ S. Chelkowski, ${ }^{2,30}$ Y. Chen, ${ }^{6}$ V. Chickarmane, ${ }^{15, i}$ D. Chin,${ }^{35}$ N. Christensen, ${ }^{8}$ D. Churches, ${ }^{7}$ T. Cokelaer, ${ }^{7}$ C. Colacino, ${ }^{32}$ R. Coldwell, ${ }^{33}$ M. Coles,${ }^{14, j}$ D. Cook,${ }^{13}$ T. Corbitt, ${ }^{12}$ D. Coyne, ${ }^{11}$ J. D. E. Creighton, ${ }^{38}$ T. D. Creighton, ${ }^{11}$ D. R. M. Crooks, ${ }^{34}$ P. Csatorday, ${ }^{12}$ B. J. Cusack, ${ }^{3}$ C. Cutler, ${ }^{1}$ E. D'Ambrosio, ${ }^{11}$ K. Danzmann, ${ }^{30,2}$ E. Daw, ${ }^{15, k}$ D. DeBra, ${ }^{25}$ T. Delker ${ }^{33,1}$ V. Dergachev, ${ }^{35}$ R. DeSalvo, ${ }^{11}$ S. Dhurandhar, ${ }^{10}$ A. Di Credico,${ }^{26}$ M. Diaz, ${ }^{28}$ H. Ding, ${ }^{11}$ R. W. P. Drever, ${ }^{4}$ R. J. Dupuis,${ }^{34}$ J. A. Edlund, ${ }^{11, b}$ P. Ehrens, ${ }^{11}$ E. J. Elliffe,,${ }^{34}$ T. Etzel, ${ }^{11}$ M. Evans,${ }^{11}$ T. Evans, ${ }^{14}$ S. Fairhurst,${ }^{38}$ C. Fallnich,${ }^{30}$ D. Farnham, ${ }^{11}$ M. M. Fejer, ${ }^{25}$ T. Findley ${ }^{24}$ M. Fine, ${ }^{11}$ L. S. Finn,,${ }^{27}$ K. Y. Franzen,${ }^{33}$ A. Freise,,${ }^{2, m}$ R. Frey,${ }^{36}$ P. Fritschel, ${ }^{12}$ V. V. Frolov, ${ }^{14}$ M. Fyffe, ${ }^{14}$ K. S. Ganezer, ${ }^{5}$ J. Garofoli, ${ }^{13}$ J. A. Giaime, ${ }^{15}$ A. Gillespie, ${ }^{11, n}$ K. Goda, ${ }^{12}$ G. González, ${ }^{15}$ S. Goßler, ${ }^{30}$ P. Grandclément, ${ }^{22, o}$ A. Grant, ${ }^{34}$ C. Gray, ${ }^{13}$ A. M. Gretarsson, ${ }^{14}$ D. Grimmett, ${ }^{11}$ H. Grote,${ }^{2}$ S. Grunewald, ${ }^{1}$ M. Guenther, ${ }^{13}$ E. Gustafson, ${ }^{25, p}$ R. Gustafson, ${ }^{35}$ W. O. Hamilton, ${ }^{15}$ M. Hammond, ${ }^{14}$ J. Hanson, ${ }^{14}$ C. Hardham, ${ }^{25}$ J. Harms,${ }^{18}$ G. Harry, ${ }^{12}$ A. Hartunian, ${ }^{11}$ J. Heefner, ${ }^{11}$ Y. Hefetz, ${ }^{12}$ G. Heinzel, ${ }^{2}$ I. S. Heng, ${ }^{30}$ M. Hennessy, ${ }^{25}$ N. Hepler, ${ }^{27}$ A. Heptonstall, ${ }^{34}$ M. Heurs, ${ }^{30}$ M. Hewitson, ${ }^{2}$ S. Hild, ${ }^{2}$ N. Hindman, ${ }^{13}$ P. Hoang, ${ }^{11}$ J. Hough, ${ }^{34}$ M. Hrynevych,${ }^{11, q}$ W. Hua,${ }^{25}$ M. Ito ${ }^{36}$ Y. Itoh, ${ }^{1}$ A. Ivanov, ${ }^{11}$ O. Jennrich,${ }^{34, r}$ B. Johnson, ${ }^{13}$ W. W. Johnson, ${ }^{15}$ W. R. Johnston,${ }^{28}$ D. I. Jones, ${ }^{27}$ L. Jones, ${ }^{11}$ D. Jungwirth, ${ }^{11, \mathrm{~s}}$ V. Kalogera, ${ }^{22}$ E. Katsavounidis,${ }^{12}$ K. Kawabe, ${ }^{13}$ S. Kawamura, ${ }^{21}$ W. Kells, ${ }^{11}$ J. Kern, ${ }^{14, t}$ A. Khan, ${ }^{14}$ S. Killbourn, ${ }^{34}$ C. J. Killow, ${ }^{34}$ C. Kim,${ }^{22}$ C. King, ${ }^{11}$ P. King, ${ }^{11}$ S. Klimenko, ${ }^{33}$ S. Koranda, ${ }^{38}$ K. Kötter, ${ }^{30}$ J. Kovalik, ${ }^{14, b}$ D. Kozak, ${ }^{11}$ B. Krishnan, ${ }^{1}$ M. Landry, ${ }^{13}$ J. Langdale, ${ }^{14}$ B. Lantz, ${ }^{25}$ R. Lawrence, ${ }^{12}$ A. Lazzarini,,${ }^{11}$ M. Lei, ${ }^{11}$ I. Leonor, ${ }^{36}$

K. Libbrecht, ${ }^{11}$ A. Libson, ${ }^{8}$ P. Lindquist, ${ }^{11}$ S. Liu, ${ }^{11}$ J. Logan, ${ }^{11, u}$ M. Lormand,${ }^{14}$ M. Lubinski ${ }^{13}$ H. Lück,${ }^{30,2}$

T. T. Lyons, ${ }^{11, \mathrm{u}}$ B. Machenschalk, ${ }^{1}$ M. MacInnis, ${ }^{12}$ M. Mageswaran, ${ }^{11}$ K. Mailand, ${ }^{11}$ W. Majid,${ }^{1, b}$ M. Malec, ${ }^{2,30}$ F. Mann, ${ }^{11}$ A. Marin, ${ }^{12, \mathrm{v}}$ S. Márka, ${ }^{11, \mathrm{w}}$ E. Maros, ${ }^{11}$ J. Mason, ${ }^{11, \mathrm{x}}$ K. Mason, ${ }^{12}$ O. Matherny, ${ }^{13}$ L. Matone, ${ }^{13}$ N. Mavalvala,${ }^{12}$ R. McCarthy, ${ }^{13}$ D.E. McClelland, ${ }^{3}$ M. McHugh, ${ }^{17}$ J. W. C. McNabb,${ }^{27}$ G. Mendell, ${ }^{13}$ R. A. Mercer, ${ }^{32}$ S. Meshkov, ${ }^{11}$ E. Messaritaki, ${ }^{38}$ C. Messenger, ${ }^{32}$ V. P. Mitrofanov, ${ }^{19}$ G. Mitselmakher, ${ }^{33}$ R. Mittleman, ${ }^{12}$ O. Miyakawa, ${ }^{11}$ S. Miyoki, ${ }^{11, y}$ S. Mohanty, ${ }^{28}$ G. Moreno, ${ }^{13}$ K. Mossavi, ${ }^{2}$ G. Mueller, ${ }^{33}$ S. Mukherjee ${ }^{28}$ P. Murray,${ }^{34}$ J. Myers, ${ }^{13}$ S. Nagano, ${ }^{2}$ T. Nash, ${ }^{11}$ R. Nayak, ${ }^{10}$ G. Newton, ${ }^{34}$ F. Nocera, ${ }^{11}$ J. S. Noel,${ }^{39}$ P. Nutzman, ${ }^{22}$ T. Olson ${ }^{23}$ B. O'Reilly, ${ }^{14}$ D. J. Ottaway, ${ }^{12}$ A. Ottewill, ${ }^{38, z}$ D. Ouimette, ${ }^{11, \mathrm{~s}}$ H. Overmier, ${ }^{14}$ B. J. Owen, ${ }^{27}$ Y. Pan, ${ }^{6}$ M. A. Papa, ${ }^{1}$ V. Parameshwaraiah, ${ }^{13}$ C. Parameswariah, ${ }^{14}$ M. Pedraza, ${ }^{11}$ S. Penn, ${ }^{9}$ M. Pitkin, ${ }^{34}$ M. Plissi, ${ }^{34}$ R. Prix,${ }^{1}$ V. Quetschke, ${ }^{33}$ F. Raab ${ }^{13}$ H. Radkins, ${ }^{13}$ R. Rahkola, ${ }^{36}$ M. Rakhmanov, ${ }^{33}$ S. R. Rao, ${ }^{11}$ K. Rawlins, ${ }^{12}$ S. Ray-Majumder,${ }^{38}$ V. Re, ${ }^{32}$ D. Redding,,${ }^{11, b}$ M. W. Regehr, ${ }^{11, b}$ T. Regimbau, ${ }^{7}$ S. Reid, ${ }^{34}$ K. T. Reilly, ${ }^{11}$ K. Reithmaier, ${ }^{11}$ D. H. Reitze, ${ }^{33}$ S. Richman, ${ }^{12,\{}$ R. Riesen, ${ }^{14}$ K. Riles, ${ }^{35}$ B. Rivera, ${ }^{13}$ A. Rizzi, ${ }^{14,1}$ D. I. Robertson, ${ }^{34}$ N. A. Robertson, ${ }^{25,34}$ L. Robison, ${ }^{11}$ S. Roddy, ${ }^{14}$ J. Rollins, ${ }^{12}$ J. D. Romano, ${ }^{7}$ J. Romie,${ }^{11}$ H. Rong ${ }^{33, n}$ D. Rose,${ }^{11}$ E. Rotthoff,${ }^{27}$ S. Rowan, ${ }^{34}$ A. Rüdiger, ${ }^{2}$ P. Russell, ${ }^{11}$ K. Ryan, ${ }^{13}$ I. Salzman, ${ }^{11}$ V. Sandberg, ${ }^{13}$ G. H. Sanders, ${ }^{11,\}}$ V. Sannibale, ${ }^{11}$ B. Sathyaprakash, ${ }^{7}$ P. R. Saulson, ${ }^{26}$ R. Savage, ${ }^{13}$ A. Sazonov, ${ }^{33}$ R. Schilling, ${ }^{2}$ K. Schlaufman, ${ }^{27}$ V. Schmidt, ${ }^{11, \sim}$ R. Schnabel, ${ }^{18}$ R. Schofield, ${ }^{36}$ B. F. Schutz,,${ }^{1,7}$ P. Schwinberg, ${ }^{13}$ S. M. Scott,${ }^{3}$ S. E. Seader, ${ }^{39}$ A. C. Searle,${ }^{3}$ B. Sears,${ }^{11}$ S. Seel, ${ }^{11}$ F. Seifert,${ }^{18}$ A. S. Sengupta, ${ }^{10}$ C. A. Shapiro, ${ }^{27}$, P. Shawhan, ${ }^{11}$ D. H. Shoemaker, ${ }^{12}$ Q.Z. Shu, ${ }^{33}$ A. Sibley, ${ }^{14}$ X. Siemens, ${ }^{38}$ L. Sievers, ${ }^{11, b}$ D. Sigg, ${ }^{13}$ A. M. Sintes, ${ }^{1,31}$ J. R. Smith, ${ }^{2}$ M. Smith, ${ }^{12}$ M. R. Smith, ${ }^{11}$ P. H. Sneddon, ${ }^{34}$ R. Spero, ${ }^{11, b}$ G. Stapfer, ${ }^{14}$ D. Steussy, ${ }^{8}$ K. A. Strain, ${ }^{34}$ D. Strom, ${ }^{36}$ A. Stuver ${ }^{27}$ T. Summerscales ${ }^{27}$ M. C. Sumner, ${ }^{11}$ P. J. Sutton, ${ }^{11}$ J. Sylvestre, ${ }^{11}$ A. Takamori, ${ }^{11}$ D. B. Tanner, ${ }^{33}$ H. Tariq, ${ }^{11}$ I. Taylor, ${ }^{7}$ R. Taylor, ${ }^{34}$ R. Taylor, ${ }^{11}$ K. A. Thorne, ${ }^{27}$ K. S. Thorne, ${ }^{6}$ M. Tibbits, ${ }^{27}$ S. Tilav, ${ }^{11,}$ M. Tinto, ${ }^{4, b}$ K. V. Tokmakov, ${ }^{19}$ C. Torres,${ }^{28}$ C. Torrie,${ }^{11}$ G. Traylor, ${ }^{14}$ W. Tyler,${ }^{11}$ D. Ugolini, ${ }^{29}$ C. Ungarelli, ${ }^{32}$ M. Vallisneri, ${ }^{6}$, M. van Putten, ${ }^{12}$ S. Vass,${ }^{11}$ A. Vecchio, ${ }^{32}$ J. Veitch, ${ }^{34}$ C. Vorvick, ${ }^{13}$ S. P. Vyachanin, ${ }^{19}$ L. Wallace, ${ }^{11}$ H. Walther, ${ }^{18}$ H. Ward ${ }^{34}$ B. Ware, ${ }^{11, b}$ K. Watts, ${ }^{14}$ D. Webber, ${ }^{11}$ A. Weidner,${ }^{18}$ U. Weiland,${ }^{30}$ A. Weinstein,,${ }^{11}$ R. Weiss,${ }^{12}$ H. Welling,${ }^{30}$ L. Wen, ${ }^{11}$ S. Wen, ${ }^{15}$ J. T. Whelan, ${ }^{17}$ S. E. Whitcomb, ${ }^{11}$ B. F. Whiting, ${ }^{33}$ S. Wiley, ${ }^{5}$ C. Wilkinson,,${ }^{13}$ P. A. Willems, ${ }^{11}$ P. R. Williams, ${ }^{1,}$ R. Williams, ${ }^{4}$ B. Willke, ${ }^{30}$ A. Wilson, ${ }^{11}$ B. J. Winjum, ${ }^{27, e}$ W. Winkler, ${ }^{2}$ S. Wise, ${ }^{33}$ A. G. Wiseman, ${ }^{38}$ 
G. Woan, ${ }^{34}$ R. Wooley, ${ }^{14}$ J. Worden, ${ }^{13}$ W. Wu,${ }^{33}$ I. Yakushin, ${ }^{14}$ H. Yamamoto, ${ }^{11}$ S. Yoshida, ${ }^{24}$ K. D. Zaleski, ${ }^{27}$ M. Zanolin, ${ }^{12}$ I. Zawischa, ${ }^{30}$ L. Zhang, ${ }^{11}$ R. Zhu, ${ }^{1}$ N. Zotov,${ }^{16}$ M. Zucker, ${ }^{14}$ and J. Zweizig ${ }^{11}$

\title{
(LIGO Scientific Collaboration)
}

\author{
${ }^{1}$ Albert-Einstein-Institut, Max-Planck-Institut für Gravitationsphysik, D-14476 Golm, Germany \\ ${ }^{2}$ Albert-Einstein-Institut, Max-Planck-Institut für Gravitationsphysik, D-30167 Hannover, Germany \\ ${ }^{3}$ Australian National University, Canberra, 0200, Australia \\ ${ }^{4}$ California Institute of Technology, Pasadena, California 91125, USA \\ ${ }^{5}$ California State University Dominguez Hills, Carson, California 90747, USA \\ ${ }^{6}$ Caltech-CaRT, Pasadena, California 91125, USA \\ ${ }^{7}$ Cardiff University, Cardiff, CF2 3YB, United Kingdom \\ ${ }^{8}$ Carleton College, Northfield, Minnesota 55057, USA \\ ${ }^{9}$ Hobart and William Smith Colleges, Geneva, New York 14456, USA \\ ${ }^{10}$ Inter-University Centre for Astronomy and Astrophysics, Pune-411007, India \\ ${ }^{11}$ LIGO-California Institute of Technology, Pasadena, California 91125, USA \\ ${ }^{12}$ LIGO-Massachusetts Institute of Technology, Cambridge, Massachusetts 02139, USA \\ ${ }^{13}$ LIGO Hanford Observatory, Richland, Washington 99352, USA \\ ${ }^{14}$ LIGO Livingston Observatory, Livingston, Louisiana 70754, USA \\ ${ }^{15}$ Louisiana State University, Baton Rouge, Louisiana 70803, USA \\ ${ }^{16}$ Louisiana Tech University, Ruston, Louisiana 71272, USA \\ ${ }^{17}$ Loyola University, New Orleans, Louisiana 70118, USA \\ ${ }^{18}$ Max Planck Institut für Quantenoptik, D-85748, Garching, Germany \\ ${ }^{19}$ Moscow State University, Moscow, 119992, Russia \\ ${ }^{20}$ NASA/Goddard Space Flight Center, Greenbelt, Maryland 20771, USA \\ ${ }^{21}$ National Astronomical Observatory of Japan, Tokyo 181-8588, Japan \\ ${ }^{22}$ Northwestern University, Evanston, Illinois 60208, USA \\ ${ }^{23}$ Salish Kootenai College, Pablo, Montana 59855, USA \\ ${ }^{24}$ Southeastern Louisiana University, Hammond, Louisiana 70402, USA \\ ${ }^{25}$ Stanford University, Stanford, California 94305, USA \\ ${ }^{26}$ Syracuse University, Syracuse, New York 13244, USA \\ ${ }^{27}$ The Pennsylvania State University, University Park, Pennsylvania 16802, USA \\ ${ }^{28}$ The University of Texas at Brownsville and Texas Southmost College, Brownsville, Texas 78520, USA \\ ${ }^{29}$ Trinity University, San Antonio, Texas 78212, USA \\ ${ }^{30}$ Universität Hannover, D-30167 Hannover, Germany \\ ${ }^{31}$ Universitat de les Illes Balears, E-07122 Palma de Mallorca, Spain \\ ${ }^{32}$ University of Birmingham, Birmingham, B15 2TT, United Kingdom \\ ${ }^{33}$ University of Florida, Gainesville, Florida 32611, USA \\ ${ }^{34}$ University of Glasgow, Glasgow, G12 8QQ, United Kingdom \\ ${ }^{35}$ University of Michigan, Ann Arbor, Michigan 48109, USA \\ ${ }^{36}$ University of Oregon, Eugene, Oregon 97403, USA \\ ${ }^{37}$ University of Rochester, Rochester, New York 14627, USA \\ ${ }^{38}$ University of Wisconsin-Milwaukee, Milwaukee, Wisconsin 53201, USA \\ ${ }^{39}$ Washington State University, Pullman, Washington 99164, USA
}

(Received 21 February 2005; published 12 August 2005)

We have performed a search for bursts of gravitational waves associated with the very bright gamma ray burst GRB030329, using the two detectors at the LIGO Hanford Observatory. Our search covered the most sensitive frequency range of the LIGO detectors (approximately $80--2048 \mathrm{~Hz}$ ), and we specifically targeted signals shorter than $\simeq 150 \mathrm{~ms}$. Our search algorithm looks for excess correlated power between the two interferometers and thus makes minimal assumptions about the gravitational waveform. We observed no candidates with gravitational-wave signal strength larger than a predetermined threshold. We report frequency-dependent upper limits on the strength of the gravitational waves associated with GRB030329. Near the most sensitive frequency region, around $\simeq 250 \mathrm{~Hz}$, our root-sum-square (RSS) gravitational-wave strain sensitivity for optimally polarized bursts was better than $h_{\mathrm{RSS}} \simeq 6 \times$ $10^{-21} \mathrm{~Hz}^{-1 / 2}$. Our result is comparable to the best published results searching for association between gravitational waves and gamma ray bursts.

DOI: 10.1103/PhysRevD.72.042002

PACS numbers: 04.80.Nn, 07.05.Kf, 95.85.Sz, 97.60.Bw 


\section{INTRODUCTION}

Gamma ray bursts (GRBs) are short but very energetic pulses of gamma rays from astrophysical sources, with duration ranging between $10 \mathrm{~ms}$ and $100 \mathrm{~s}$. GRBs are historically divided into two classes [1,2] based on their duration: "short" $(<2 \mathrm{~s})$ and "long" $(>2 \mathrm{~s})$. Both classes are isotropically distributed and their detection rate can be as large as one event per day. The present consensus is that long GRBs [2] are the result of the core collapse of massive stars resulting in black hole formation. The violent formation of black holes has long been proposed as a potential source of gravitational waves. Therefore, we have reason to expect strong association between GRBs and gravitational waves [3-5]. In this paper, we

\footnotetext{
${ }^{\text {a}}$ Present address: Stanford Linear Accelerator Center.

${ }^{\mathrm{b}}$ Present address: Jet Propulsion Laboratory.

${ }^{\mathrm{c}}$ Permanent address: HP Laboratories.

${ }^{\mathrm{d}}$ Present address: Rutherford Appleton Laboratory.

${ }^{\mathrm{e}}$ Present address: University of CA, Los Angeles.

${ }^{\mathrm{f}}$ Present address: Hofstra University.

${ }^{\text {g}}$ Permanent address: GReCO, Institut d'Astrophysique de Paris (CNRS).

${ }^{\mathrm{h}}$ Present address: La Trobe University, Bundoora VIC, Australia.

${ }^{\mathrm{i}}$ Present address: Keck Graduate Institute.

${ }^{\mathrm{j}}$ Present address: National Science Foundation.

${ }^{\mathrm{k}}$ Present address: University of Sheffield.

${ }^{1}$ Present address: Ball Aerospace Corporation.

${ }^{\mathrm{m}}$ Present address: European Gravitational Observatory.

${ }^{\mathrm{n}}$ Present address: Intel Corp.

${ }^{\mathrm{o}}$ Present address: University of Tours, France.

${ }^{\mathrm{p}}$ Present address: Lightconnect Inc.

${ }^{\mathrm{q}}$ Present address: W.M. Keck Observatory.

${ }^{\mathrm{r}}$ Present address: ESA Science and Technology Center.

${ }^{\text {s}}$ Present address: Raytheon Corporation.

${ }^{\mathrm{t}}$ Present address: NM Institute of Mining and Technology /

Magdalena Ridge Observatory Interferometer.

uresent address: Mission Research Corporation.

${ }^{\mathrm{v}}$ Present address: Harvard University.

${ }^{\mathrm{w}}$ Permanent address: Columbia University.

${ }^{x}$ Present address: Lockheed-Martin Corporation.

${ }^{\mathrm{y}}$ Permanent address: University of Tokyo, Institute for Cosmic Ray Research.

${ }^{\mathrm{z}}$ Permanent address: University College Dublin.

${ }^{\text {aa }}$ Present address: Research Electro-Optics Inc.

${ }^{\mathrm{ab}}$ Present address: Institute of Advanced Physics, Baton Rouge, LA.

${ }^{\text {ac }}$ Present address: Thirty Meter Telescope Project at Caltech.

${ }^{\mathrm{ad}}$ Present address: European Commission, DG Research, Brussels, Belgium.

${ }^{\mathrm{ae}}$ Present address: University of Chicago.

${ }^{a f}$ Present address: LightBit Corporation.

${ }^{\text {ag }}$ Permanent address: IBM Canada Ltd.

${ }^{\text {ah }}$ Present address: University of DE.

${ }^{a i}$ Permanent address: Jet Propulsion Laboratory.

${ }^{\text {aj }}$ Present address: Shanghai Astronomical Observatory.

${ }^{a k}$ Present address: Laser Zentrum Hannover.

${ }^{a l}$ Electronic address: http://www.ligo.org
}

report on a search for a possible short burst of gravitational waves associated with GRB030329 using data collected by the Laser Interferometer Gravitational-Wave Observatory (LIGO).

On March 29, 2003, instrumentation aboard the HETE-2 satellite [6] detected a very bright GRB, designated GRB030329. The GRB was followed by a bright and well-measured afterglow from which a redshift [7] of $z=$ 0.1685 (distance $\simeq 800 \mathrm{Mpc}$ [8]) was determined. After approximately 10 days, the afterglow faded to reveal an underlying supernova (SN) spectrum, SN2003dh [9]. This GRB is the best studied to date, and confirms the link between long GRBs and supernovae.

At the time of GRB030329, LIGO was engaged in a 2month long data run. The LIGO detector array consists of three interferometers, two at the Hanford, WA site and one at the Livingston, LA site. Unfortunately, the Livingston interferometer was not operating at the time of the GRB; therefore, the results presented here are based on the data from only the two Hanford interferometers. The LIGO detectors are still undergoing commissioning, but at the time of GRB030329, their sensitivity over the frequency band 80 to $2048 \mathrm{~Hz}$ exceeded that of any previous gravitational-wave search, with the lowest strain noise of $\simeq 6 \times 10^{-22} \mathrm{~Hz}^{-1 / 2}$ around $250 \mathrm{~Hz}$.

A number of long GRBs have been associated with $\mathrm{x}$-ray, radio and/or optical afterglows, and the cosmological origin of the host galaxies of their afterglows has been unambiguously established by their observed redshifts, which are of order unity [2]. The smallest observed redshift of an optical afterglow associated with a detected GRB (GRB980425 [10-12]) is $z=0.0085$ ( $\simeq 35 \mathrm{Mpc}$ ). GRB emissions are very likely strongly beamed [13,14], a factor that affects estimates of the energy released in gamma rays (a few times $10^{50} \mathrm{erg}$ ), and their local true event rate (about 1 per year within a distance of $100 \mathrm{Mpc}$ ).

In this search, we have chosen to look for a burst of gravitational waves in a model independent way. Corecollapse [4], black hole formation [5,15] and black hole ringdown $[16,17]$ may each produce gravitational-wave emissions, but there are no accurate or comprehensive predictions describing the gravitational-wave signals that might be associated with GRB type sources. Thus, a traditional matched filtering approach $[18,19]$ is not possible in this case. To circumvent the uncertainties in the waveforms, our algorithm does not presume any detailed knowledge of the gravitational waveform and we only apply general bounds on the waveform parameters. Based on current theoretical considerations, we anticipate the signals in our detectors to be weak, comparable to or less than the detector's noise [20-22].

This paper is organized as follows: Section II summarizes the currently favored theories of GRBs and their consequences for gravitational-wave detection. Section III provides observational details pertinent to 
GRB030329. Section IV briefly describes the LIGO detectors and their data. Section V discusses the method of analysis of the LIGO data. In Section VI we compare the events in the signal region with expectations and we use simulated signal waveforms to determine detection efficiencies. We also present and interpret the results in this section. Section VII offers a comparison with previous analyses, a conclusion, and an outlook for future searches of this type.

\section{PRODUCTION OF GRAVITATIONAL WAVES IN MASSIVE CORE COLLAPSES}

The apparent spatial association of GRB afterglows with spiral arms, and by implication star formation regions in remote galaxies, has lead to the current "collapsar" or "hypernova" scenario $[23,24]$ in which the collapse of a rotating, massive star to a Kerr black hole can lead to relativistic ejecta emitted along a rotation axis and the associated production of a GRB jet. The identification of GRB030329 with the supernova SN2003dh (Sec. III below) gives further support to this association. This observation is consistent with the theory that the GRB itself is produced by an ultrarelativistic jet associated with a central black hole. Stellar mass black holes in supernovae must come from more massive stars. Reference [24] presents "maps" in the metallicity-progenitor mass plane of the end states of stellar evolution and shows that progenitors with $25 M_{\odot}$ can produce black holes by fallback accretion.

The observed pulsar kick velocities of $\simeq 500 \mathrm{~km} / \mathrm{s}$ hint at a strong asymmetry around the time of maximum compression, which may indicate deviations from spherical symmetry in the progenitor. The resulting back reaction on the core from the neutrino heating provides yet another potential physical mechanism for generating a gravitational-wave signal. In the model of [25] it imparts a kick of $400-600 \mathrm{~km} / \mathrm{s}$ and an induced gravitational-wave strain roughly an order of magnitude larger than in [20] and an order of magnitude smaller than [26].

Theoretical work on gravitational-wave (GW) signals in the process of core collapse in massive stars has advanced much in recent years, but still does not provide detailed waveforms. Current models take advantage of the increase in computational power and more sophisticated input physics to include both 2D and 3D calculations, utilizing realistic precollapse core models and a detailed, complex equation of state of supernovae that produce neutron stars. The most recent studies by independent groups give predictions for the strain amplitude within a similar range, despite the fact that the dominant physical mechanisms for gravitational-wave emission in these studies are different [20,21,26-28]. The calculations of [20] are qualitatively different from previous core-collapse simulations in that the dominant contribution to the gravitational-wave signal is neutrino-driven convection, about 20 times larger than the axisymmetric core-bounce gravitational-wave signal.
The applicability of the above models to GRBs is not clear, since the model end points are generally neutron stars, rather than black holes. Another recent model involves accretion disks around Kerr black holes [29], subject to nonaxisymmetric Papaloizou-Pringle instabilities [30] in which an acoustic wave propagates toroidally within the fallback material. They are very interesting since they predict much higher amplitudes for the gravitational-wave emission.

For our search, the main conclusion to draw is that in spite of the dramatic improvement in the theoretical models, there are no gravitational waveforms that could be reliably used as templates for a matched filter search, and that any search for gravitational waves should ideally be as waveform independent as practical. Conversely, detection of gravitational waves associated with a GRB would almost certainly provide crucial new input for GRB/SN astrophysics. It is also clear that the predictions of gravitational-wave amplitudes are uncertain by several orders of magnitude, making it difficult to predict the probability to observe the gravitational-wave signature of distant GRBs.

The timeliness of searching for a gravitational-wave signal associated with GRBs is keen in light of the recent work by [20,21]. Reference [20] finds that the signal due to neutrino convection exceeds that due to the core bounce and therefore a chaotic signal would be expected. Studies with simplified or no neutrino transport (e.g., [21,22]) find the core bounce to be the dominant contributor to the GW signal. The large-scale, coherent mass motions involved in the core bounce leads to a predicted gravitational-wave signal resembling a damped sinusoid.

\section{GRB030329 RELATED OBSERVATIONAL RESULTS}

\section{A. Discovery of GRB 030329 and its afterglow}

On March 29, 2003 at 11:37:14.67 UTC, a GRB triggered the FREGATE instrument on board the HETE-2 satellite [6,31-33]. The GRB had an effective duration of $\simeq 50 \mathrm{~s}$, and a fluence of $1.08 \times 10^{-4} \mathrm{erg} / \mathrm{cm}^{2}$ in the $30-$ $400 \mathrm{keV}$ band [33]. The KONUS detector on board the Wind satellite also detected it [34], triggering about $15 \mathrm{sec}$ after HETE-2. KONUS observed the GRB for about $35 \mathrm{sec}$, and measured a fluence of $1.6 \times 10^{-4} \mathrm{erg} / \mathrm{cm}^{2}$ in the $15-$ $5000 \mathrm{keV}$ band. The measured gamma ray fluences place this burst among the brightest GRBs. Figure 1 shows the HETE-2 light curve for GRB030329 [35].

The rapid localization of the GRB by HETE ground analysis gave an accurate position which was distributed about 73 min after the original trigger. A few hours later, an optical afterglow [7,36] was discovered with magnitude $R=12.4$, making it the brightest optical counterpart to any GRB detected to date. The RXTE [37] satellite measured a $x$-ray flux of $1.4 \times 10^{-10} \mathrm{erg} \mathrm{s}^{-1} \mathrm{~cm}^{-2}$ in the $2-10 \mathrm{keV}$ band about $4 \mathrm{~h} 51 \mathrm{~m}$ after the HETE trigger, making this 


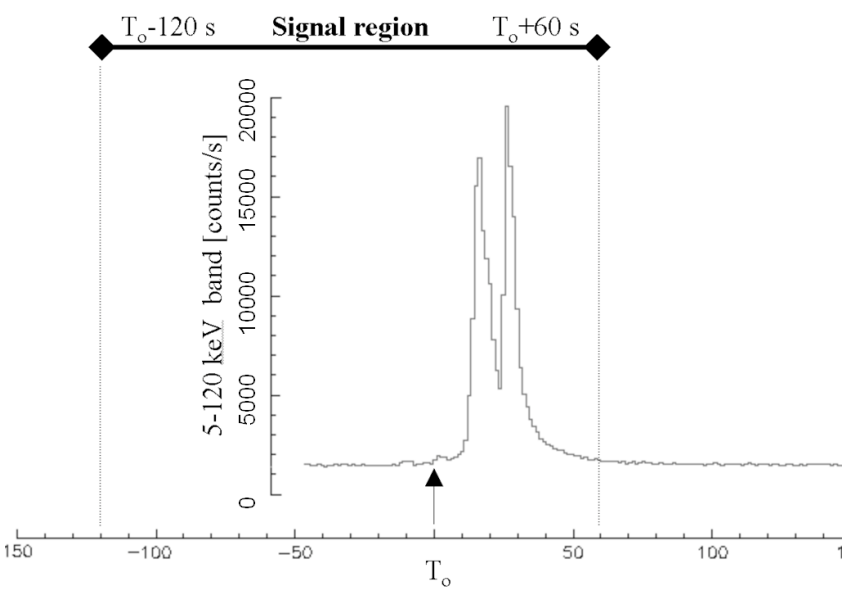

Time from GRB030329 Fregate_B trigger $\left(\mathrm{T}_{\mathrm{o}}=732973047.7\right)[\mathrm{s}]$

FIG. 1. The GRB030329 light curve as measured by the HETE-2 FREGATE B instrument. The arrow indicates the HETE trigger time. The signal region analyzed in this study is indicated by the horizontal bar at the top. This figure is the courtesy of the HETE Collaboration.

one of the brightest x-ray afterglows detected by RXTE [38]. The National Radio Astronomy Observatory (NRAO) observed [39] the radio afterglow, which was the brightest radio afterglow detected to date [40]. Spectroscopic measurements of the bright optical afterglow [41] revealed emission and absorption lines, and the inferred redshift $\left(z=0.1685\right.$, luminosity distance $\left.D_{L} \cong 800 \mathrm{Mpc}\right)$ made this the second nearest GRB with a measured distance. To date, no host galaxy has been identified. It is likely that numerous other GRBs have been closer than GRB030329, but the lack of identified optical counterparts has left their distances undetermined.

Spectroscopic measurements $[8,42,43]$, about a week after the GRB trigger, revealed evidence of a supernova spectrum emerging from the light of the bright optical afterglow, which was designated SN2003dh. The emerging supernova spectrum was similar to the spectrum of SN1998bw a week before its brightness maximum [44,45].

SN1998bw was a supernova that has been spatially and temporally associated with GRB980425 [10-12], and was located in a spiral arm of the barred spiral galaxy ESO 184G82 at a redshift of $z=0.0085$ ( $\simeq 35 \mathrm{Mpc}$ ), making it the nearest GRB with a measured distance. The observed spectra of SN2003dh and SN1998bw, with their lack of hydrogen and helium features, place them in the Type Ic supernova class. These observations, together with the observations linking GRB980425 (which had a duration of $\simeq 23 \mathrm{~s}$ ) to SN1998bw, make the case that collapsars are progenitors for long GRBs more convincing. In the case of SN1998bw, Woosley et al. [46] and Iwamoto et al. [11] found that its observed optical properties can be well modeled by the core collapse of a $\mathrm{C}+\mathrm{O}$ core of mass $6 M_{\odot}$ (main sequence mass of $25 M_{\odot}$ ) with a kinetic energy of $\simeq 2 \times 10^{52}$ ergs. This energy release is about an order of magnitude larger than the energies associated with typical supernovae.

\section{B. GRB030329 energetics}

A widely used albeit naive quantity to describe the energy emitted by GRBs is the total isotropic equivalent energy in gamma rays:

$$
E_{\text {iso }}=4 \pi(B C) D_{L}^{2} f /(1+z) \approx 2 \times 10^{52} \mathrm{erg}
$$

where $f$ is the measured fluence in the HETE- 2 waveband and $\mathrm{BC}$ is the approximate bolometric correction for HETE-2 for long GRBs. Using a "band spectrum" [47] with a single power law to model the gamma ray spectrum, and using a spectral index, $\beta=-2.5$, gives that the GRB's total energy integrated from 1 to $5 \mathrm{GeV}$ is greater than that present in the band $30-400 \mathrm{keV}$ by a factor 2.2 .

However, it is generally believed that GRBs are strongly beamed, and that the change in slope in the afterglow light curve corresponds to the time when enough deceleration has occurred so that relativistic beaming is diminished to the point at which we "see" the edge of the jet. This occurs during the time in which the relativistic ejecta associated with the GRB plows through the interstellar medium, and the beaming factor $\Gamma^{-1}$, where $\Gamma$ is the bulk Lorentz factor of the flow, increases from a value smaller than the beaming angle $\Theta_{j}$, to a value larger than $\Theta_{j}$. Effectively, prior to this time the relativistic ejecta appears to be part of a spherical expansion, the edges of which cannot be seen because they are outside of the beam, while after this time the observer perceives a jet of finite width.

This leads to a faster decline in the light curves. Zeh $e t$ al. and $\mathrm{Li}$ et al. [48,49] show that the initial "break" or strong steepness in the light curve occurs at about $10 \mathrm{~h}$ after the initial HETE-2 detection.

Frail et al. [13] give a parametric relation between beaming angle $\Theta_{j}$, break time $t_{j}$, and $E_{\text {iso }}$ as

$$
\begin{aligned}
\Theta_{j} \approx & 0.057\left(\frac{t_{j}}{24 \mathrm{~h}}\right)^{3 / 8}\left(\frac{1+z}{2}\right)^{-3 / 8}\left(\frac{E_{\mathrm{iso}}}{10^{53} \mathrm{ergs}}\right)^{-1 / 8}\left(\frac{\eta_{\gamma}}{0.2}\right)^{1 / 8} \\
& \times\left(\frac{n}{0.1 \mathrm{~cm}^{-3}}\right)^{1 / 8},
\end{aligned}
$$

where $\Theta_{j}$ is measured in radians. It was argued that the fireball converts the energy in the ejecta into gamma rays efficiently [50] $\left(\eta_{\gamma} \approx 0.2\right)$, and that the mean circumburst density is $n \approx 0.1 \mathrm{~cm}^{-3}$ [51]. Evaluating Eq. (3.2) for the parameters of GRB030329 $\left(t_{j} \approx 10 \mathrm{~h}, z=0.1685\right.$, and $E_{\text {iso }}=2 \times 10^{52} \mathrm{erg}$ ) gives $\Theta_{j} \approx 0.07 \mathrm{rad}$.

Therefore the beaming factor that relates the actual energy released in gamma rays $\left(E_{\gamma}\right)$ to the isotropic equivalent energy is $\Theta_{j}^{2} / 2 \approx 1 / 400$, so that $E_{\gamma} \approx$ $5 \times 10^{49} \mathrm{erg}$. Comparing $E_{\text {iso }}$ and $E_{\gamma}$ with the histograms in Fig. 2 of Frail et al. [13], GRB030329 resides at the lower end of the energy distributions. The calculated iso- 


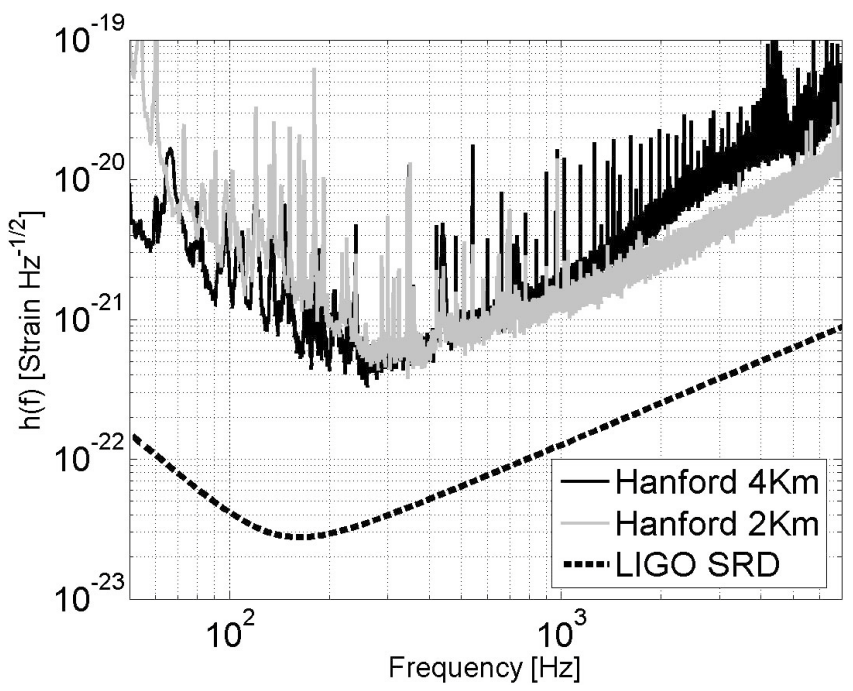

FIG. 2. Typical LIGO Hanford sensitivity curves during the S2 Run [strain $\mathrm{Hz}^{-1 / 2}$ ] (black and gray lines). The LIGO design sensitivity goal (SRD) is also indicated (dashed line).

tropic energy from GRB980425, the GRB associated with SN1998bw, is also low ( $\left.\simeq 10^{48} \mathrm{erg}\right)$.

\section{OVERVIEW OF THE LIGO DETECTORS}

The three LIGO detectors are orthogonal arm Michelson laser interferometers, aiming to detect gravitational waves by interferometrically monitoring the relative (differential) separation of mirrors, which play the role of test masses. The LIGO Hanford Observatory (LHO) operates two identically oriented interferometric detectors, which share a common vacuum envelope: one having $4 \mathrm{~km}$ long arms (H1), and one having $2 \mathrm{~km}$ long arms (H2). The LIGO Livingston Observatory operates a single $4 \mathrm{~km}$ long detector (L1). The two sites are separated by $\simeq 3000 \mathrm{~km}$, representing a maximum arrival time difference of $\simeq \pm 10 \mathrm{~ms}$.

A complete description of the LIGO interferometers as they were configured during LIGO's first science run (S1) can be found in Ref. [52].

\section{A. Detector calibration and configuration}

To calibrate the error signal, the response to a known differential arm strain is measured, and the frequencydependent effect of the feedback loop gain is measured and compensated for. During detector operation, changes in calibration are tracked by injecting continuous, fixedamplitude sinusoidal excitations into the end test mass control systems, and monitoring the amplitude of these signals at the measurement (error) point. Calibration uncertainties at the Hanford detectors were estimated to be $<11 \%$.

Significant improvements were made to the LIGO detectors following the S1 run, held in early fall of 2002:
(1) The analog suspension controllers on the $\mathrm{H} 2$ and L1 interferometers were replaced with digital suspension controllers of the type installed on H1 during S1, resulting in lower electronics noise.

(2) The noise from the optical lever servo that damps the angular excitations of the interferometer optics was reduced.

(3) The wave front sensing system for the H1 interferometer was used to control 8 of 10 alignment degrees of freedom for the main interferometer. As a result, it maintained a much more uniform operating point over the run.

(4) The high frequency sensitivity was improved by operating the interferometers with higher effective power, about $1.5 \mathrm{~W}$.

These changes led to a significant improvement in detector sensitivity. Figure 2 shows typical spectra achieved by the LIGO interferometers during the $\mathrm{S} 2$ run. The differences among the three LIGO spectra reflect differences in the operating parameters and hardware implementations of the three instruments which are in various stages of reaching the final design configuration.

\section{B. The second science run}

The data analyzed in this paper were taken during LIGO's second science run (S2), which spanned approximately 60 days from February 14 to April 14, 2003. During this time, operators and scientific monitors attempted to maintain continuous low noise operation. The duty cycle for the interferometers, defined as the fraction of the total run time when the interferometer was locked and in its low noise configuration, was approximately $74 \%$ for $\mathrm{H} 1$ and $58 \%$ for $\mathrm{H} 2$. The longest continuous locked stretch for any interferometer during S2 was 66 hours for H1.

At the time of the GRB030329 both Hanford interferometers were locked and taking science mode data. For this analysis we relied on the single, $\simeq 4.5 \mathrm{~h}$ long coincident lock stretch, which started $\simeq 3.5 \mathrm{~h}$ before the trigger time. With the exception of the signal region, we utilized $\simeq 98 \%$ of the data within this lock stretch as the background region (defined in Sec. V). $60 \mathrm{sec}$ of data before and after the signal region were not included in the background region. Data from the beginning and from the end of the lock stretch were not included in the background region to avoid using possibly nonstationary data, which might be associated with these regions.

As described below, the false alarm rate estimate, based on background data, must be applicable to the data within the signal region. We made a conservative choice and avoided using background data outside of the lock stretch containing the GRB trigger time. This is important when considering the present nonstationary behavior of the interferometric detectors. 


\section{ANALYSIS}

The goal of the analysis is either to identify significant events in the signal region or, in the absence of significant events, to set a limit on the strength of the associated gravitational-wave signal. Simulations and background data were used to determine the detection efficiency for various ad hoc and model-based waveforms (Sec. VIB) and the false alarm rate of the detection algorithm, respectively.

The analysis takes advantage of the information provided by the astrophysical trigger. The trigger time determined when to perform the analysis. As discussed below, the time window to be analyzed around the trigger time was chosen to accommodate most current theoretical predictions and timing uncertainties. The source direction was needed to calculate the attenuation due to the LIGO detector's antenna pattern for the astrophysical interpretation.

The two colocated and coaligned Hanford detectors had very similar frequency-dependent response functions at the time of the trigger. Consequently, the detected arrival time and recorded waveforms of a gravitational-wave signal should be essentially the same in both detectors. It is natural then to consider cross correlation of the two data streams as the basis of a search algorithm. This conclusion can also be reached via a more formal argument based on the maximum log-likelihood ratio test $[53,54]$.

The schematic of the full analysis pipeline is shown in Fig. 3. The underlying analysis algorithm is described in detail in Ref. [54]. The background data, the signal region data and the simulations are all processed identically. The background region consists of the data where we do not expect to have a gravitational-wave signal associated with the GRB. We scan the background to determine the false alarm distribution and to set a threshold on the event strength that will yield an acceptable false alarm rate. This threshold is used when scanning the signal region

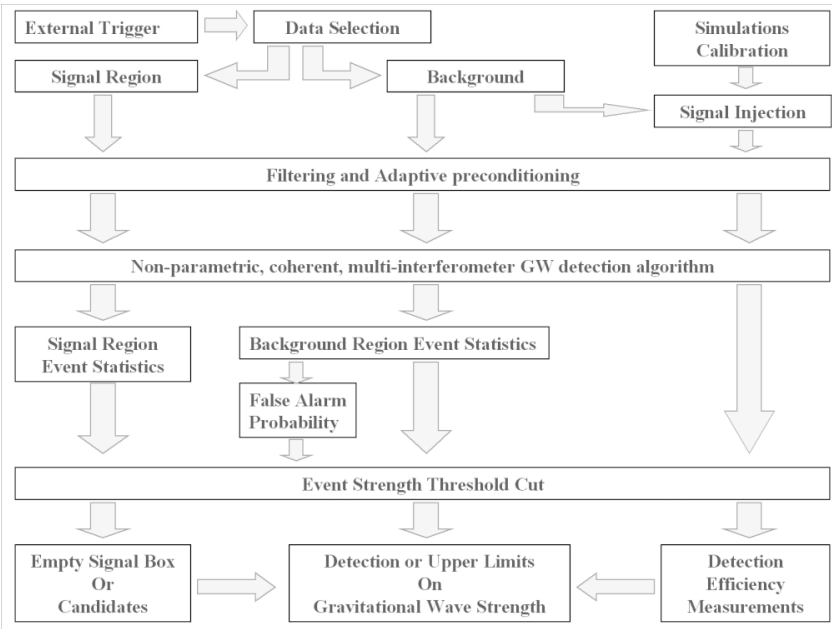

FIG. 3. The schematic of the analysis pipeline. and simulations. In order to estimate our sensitivity to gravitational waves, simulated signals of varying strength are added to the detector data streams.

The signal region around the GRB trigger is scanned to identify outstanding signals. If events were detected above threshold, in this region, their properties would be tested against those expected from gravitational waves. If no events were found above threshold, we would use the estimated sensitivity to set an upper limit on the gravitational-wave strain at the detector.

The output from each interferometer is divided into $330 \mathrm{sec}$ long segments with a $15 \mathrm{sec}$ overlap between consecutive segments (both ends), providing a tiling of the data with $300 \mathrm{sec}$ long segments. In order to avoid edge effects, the $180 \mathrm{sec}$ long signal region lies in the middle of one such $300 \mathrm{sec}$ long segment. This tiling method also allows for adaptive data conditioning and places the conditioning filter (see Sec. V B 1 below) transients well outside of the $300 \mathrm{sec}$ long segment containing the signal region.

\section{A. Choice of signal region}

Current models suggest [2] that the gravitational-wave signature should appear close to the GRB trigger time. We conservatively chose the duration and position of the signal region to over-cover most predictions and to allow for the expected uncertainties associated with the GRB trigger timing. A $180 \mathrm{sec}$ long window (see Fig. 1), starting $120 \mathrm{sec}$ before the GRB trigger time is sufficient; roughly 10 times wider than the GRB light curve features, and wide enough to include most astrophysical predictions. Most models favor an ordering where the arrival of the gravitational wave precedes the GRB trigger [2], but in a few other cases the gravitational-wave arrival is predicted to be contemporaneous $[5,55]$ to the arrival and duration of the gamma rays (i.e. after the GRB trigger). The $60 \mathrm{sec}$ region after the GRB trigger time, is sufficient to cover these predictions and also contains allowance for up to $30 \mathrm{sec}$ uncertainty on trigger timing, which is a reasonable choice in the context of the HETE light curve. Figure 1 shows a signal rise time of order $10 \mathrm{~s}$, precursor signals separated from the main peak, and significant structure within the main signal itself. Effects due to the beaming dynamics of the GRB and the instrumental definition of the trigger time can also be significant contributors to the timing uncertainty.

\section{B. Search algorithm}

\section{Data conditioning}

The data-conditioning step was designed to remove instrumental artifacts from the data streams. We used an identical data-conditioning procedure when processing the background, the signal region and the simulations. 
The raw data streams have narrowband lines, associated with the power line harmonics at multiples of $60 \mathrm{~Hz}$, the violin modes of the mirror suspension wires and other narrow band noise sources. The presence of lines has a detrimental effect on our sensitivity because lines can produce spurious correlations between detectors. In addition, the broadband noise shows significant variations over time scales of hours and smaller variations over time scales of minutes and seconds due to alignment drift and fluctuations. The background data must portray a representative sample of the detector behavior around the time of the trigger. Broadband nonstationarity can limit the duration of this useful background data and hence the reliability of our estimated false alarm rate.

Our cross-correlation-based algorithm performs best on white spectra without line features. We use notch filters to remove the well-known lines, such as power line and violin mode harmonics from both data streams. Strong lines of unknown origin with stationary mean frequency are also removed at this point. We also apply a small correction to mitigate the difference between the phase and amplitude response of the two Hanford detectors.

We bandpass filter and decimate the data to a sampling rate of $4096 \mathrm{~Hz}$ to restrict the frequency content to the $\simeq 80$ to $\simeq 2048 \mathrm{~Hz}$ region, which was the most sensitive band for both LIGO Hanford detectors during the S2 run.

In order to properly remove weaker stationary lines and the small residuals of notched strong lines, correct for small slow changes in the spectral sensitivity and whiten the spectrum of the data, we use adaptive line removal and whitening. As all strong lines are removed before the adaptive whitening, we avoid potential problems due to nonstationary lines and enhance the efficiency of the follow-up adaptive filtering stage. The conditioned data has a consistent white spectrum without major lines and sufficient stationarity, from segment to segment, throughout the background and signal regions.

The end result of the preprocessing is a data segment with a flat power spectral density (white noise), between $\simeq 80$ and $\simeq 2048 \mathrm{~Hz}$. The data conditioning was applied consistently after the signal injections. This ensures that any change in detection efficiency due to the preprocessing is properly taken into account.

\section{Gravitational wave search algorithm}

The test statistics for a pair of data streams are constructed as follows. We take pairs of short segments, one from each stretch, and compute their cross-correlation function. The actual form of the cross correlation used $\left(\tilde{C}_{k, p, j}^{m, n}\right)$ is identical to the common Euclidean inner product:

$$
\tilde{C}_{k, p, j}^{m, n}=\sum_{i=-j}^{j} H_{m}[k+i] H_{n}[k+p+i]
$$

where the preconditioned time series from detector " $x$ " is $\mathbf{H}_{x}=\left\{H_{x}[0], H_{x}[1], \ldots\right\}$ and $i, k, p$ and $j$ are all integers indexing the data time series, with each datum being $(1 / 4096) \mathrm{s}$ long. As we now only consider the two Hanford detectors " $m$ " and " $n$ " can only assume values of $1\left(H_{1}\right)$ or $2\left(H_{2}\right)$. There are therefore three free parameters to scan when searching for coherent segments of data between a pair of interferometers $(m, n)$ : (i) the center time of the segment from the first detector $(k)$; (ii) the relative time lag between the segments from the two detectors $(p)$; and (iii) the common duration of segments $(2 j+1)$ called the integration length.

The optimum integration length to use for computing the cross correlation depends on the duration of the signal and its signal-to-noise ratio, neither of which is a priori known. Therefore the cross correlation should be computed from segment pairs with start times and lengths varying over values, which should, respectively, cover the expected arrival times (signal region) and consider durations of the gravitational-wave burst signals [20,21,25-28] [ $O(1-128 \mathrm{~ms})]$.

Hence we apply a search algorithm [54] that processes the data in the following way.

(1) A three dimensional quantity $\left(C_{k, j}[p]\right)$ is constructed:

$$
C_{k, j}[p]=\left[\left(\tilde{C}_{k, p, j}^{1,2}\right)^{2}+\left(\tilde{C}_{k,-p, j}^{2,1}\right)^{2}\right]^{1 / 2},
$$

scanning the range of segment center times $(k)$, integration lengths $(2 j+1)$ and relative time shifts $(p=$ $0, \pm 1, \pm 2, \ldots)$. A coherent and coincident signal is expected to leave its localized signature within this three dimensional quantity.

We use a fine rectangular grid in relative time shift $(p)$ and integration length $(2 j+1)$ space. The spacing between grid points is $\simeq 1 \mathrm{~ms}$ for the segment center time $(k)$ and $(1 / 4096) \mathrm{s}$ for the relative time shift. The spacing of the integration lengths is approximately logarithmic. Each consecutive integration length is $\simeq 50 \%$ longer than the previous one, covering integration lengths from $\simeq 1$ to $\simeq 128 \mathrm{~ms}$.

Introducing small, nonphysical relative time shifts (much larger than the expected signal duration) between the two data streams before computing the crosscorrelation matrix suppresses the average contribution from a GW signal. This property can be used to estimate the local noise properties, thereby mitigating the effects of nonstationarity in the interferometer outputs. Accordingly, $C_{k, j}[p]$ contains the autocorrelation of the coherent signal for relative time shifts at and near $p=0$ (called "core"), while far away, in the "side lobes," the contribution from the signal autocorrelation is absent, sampling only the random contributions to the cross correlation arising from the noise. The optimal choice of the core size depends on the expected signal duration (integration length), the underlying detector noise and it cannot be smaller than 
the relative phase uncertainty of the data streams. The core region can reach as far as $5 \mathrm{~ms}$, as it increases with increasing integration length. The size of each side lobe is twice the size of the core region and the median time shift associated with the side lobes can be as large as $325 \mathrm{~ms}$ as it is also increasing with increasing integration length. We use the side lobes of $C_{k, j}[p]$ to estimate the mean $\left(\hat{\mu}_{k, j}\right)$ and variance $\left(\hat{\sigma}_{k, j}\right)$ of the local noise distribution, which is also useful in countering the effects of nonstationarity.

(2) The three dimensional quantity is reduced to a two dimensional image (see Fig. 4), called a corrgram, as follows. The values of $C_{k, j}[p]$ in the core region are standardized by subtracting $\hat{\mu}_{k, j}$ and then dividing by $\hat{\sigma}_{k, j}$. Positive standardized values in the core region are summed

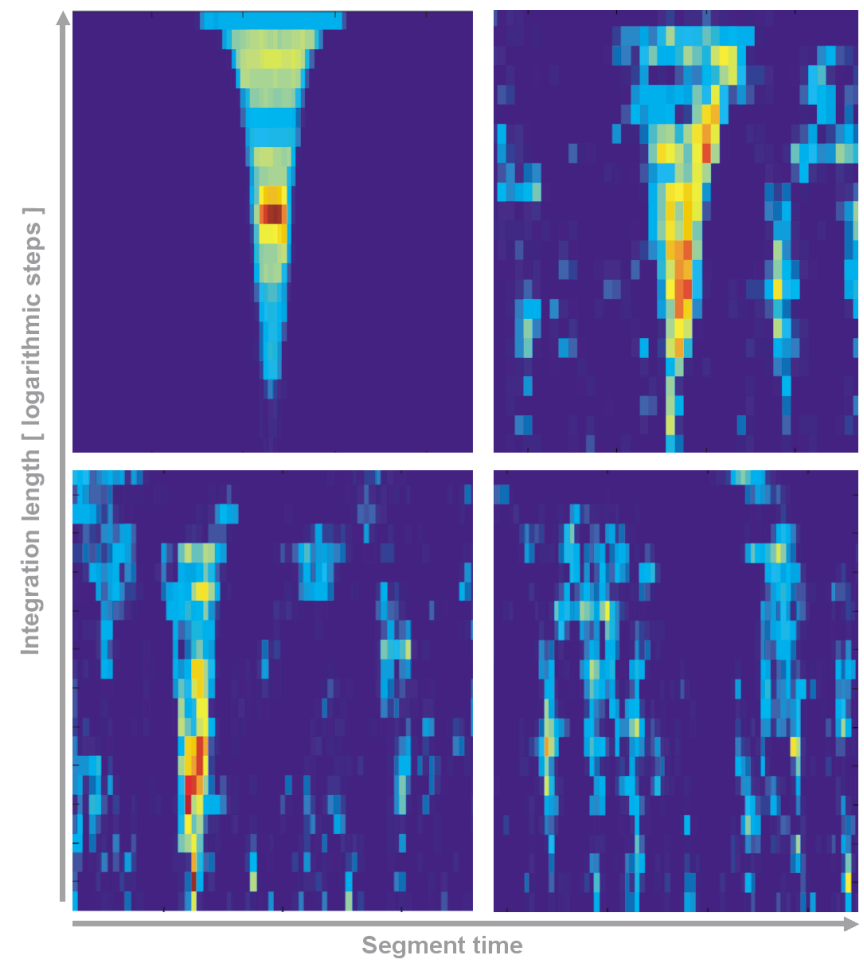

FIG. 4 (color). Examples of corrgram images. The horizontal axes are time (linearly scaled) and the vertical axes are integration length (logarithmically scaled). The color axis, an indicator of the excess correlation, is independently autoscaled for each quadrant for better visibility, therefore the meaning of colors differ from quadrant to quadrant. The time ticks also change from quadrant to quadrant for better visibility. The rainbow type color scale goes from blue to red, dark red marking the most significant points within a quadrant. The upper two quadrants show the corrgram image of injected sine Gaussians $(250 \mathrm{~Hz}$, $Q=8.9$ ). The bottom quadrants are examples of noise. The maximum of the intensity scale is significantly higher for both quadrants with injections, when compared to the noise examples. The top left injection is strong enough to be significantly above the preset detection threshold, while the top right injection is weak enough to fall significantly below the detection threshold. over $p$ to determine the value of the corrgram pixel. Each pixel is a measure of the excess cross correlation in the core region when compared to the expected distribution characterized by the side lobes for the given $(k, j)$ combination.

(3) A list of events is found by recursively identifying and characterizing significant regions (called "clusters") in the corrgram image. Each event is described by its arrival time, its optimal integration length and its strength (ES). The event's arrival time and its optimal integration length correspond to the most significant pixel of the cluster. The event strength is determined by averaging the five most significant pixels of the cluster, as this is helpful in discriminating against random fluctuations of the background noise.

The strength of each event is then compared to a preset detection threshold corresponding to the desired false alarm rate. This detection threshold is determined via extensive scans of the background region.

\section{RESULTS}

\section{A. False alarm rate measurements}

In order to assess the significance of the cross-correlated power of an event, we determined the false alarm rate versus event strength distribution. We used the full background data stretch for this measurement.

Figure 5 shows the event rate as a function of the event strength threshold for the background region. The error bars reflect $90 \%$ C.L. Poisson errors, based on the number of events within the given bin. We used this distribution to

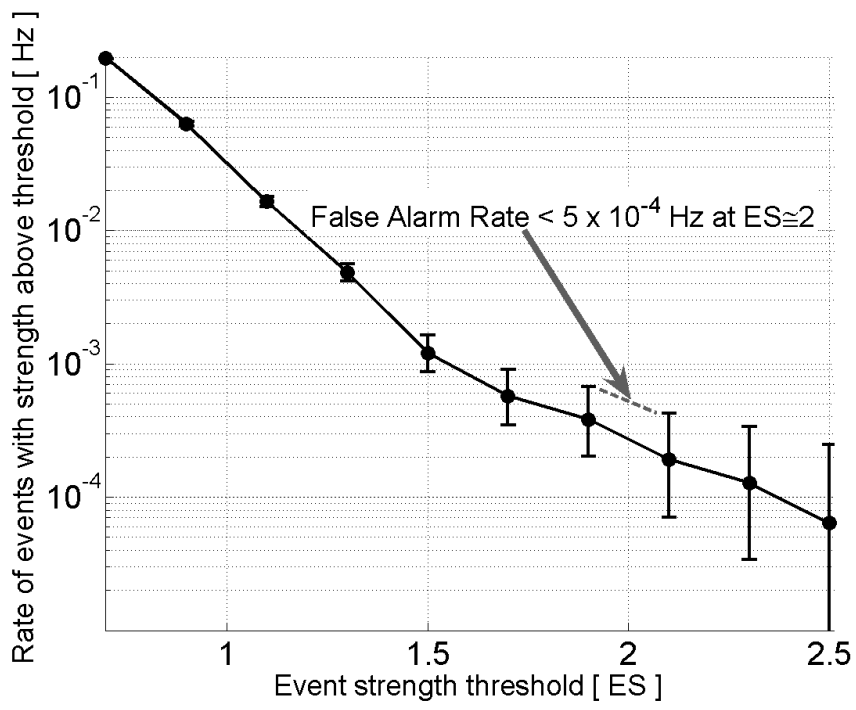

FIG. 5. False alarm rate as a function of the event strength threshold as determined from background data. The error bars reflect $90 \%$ C.L. Poisson errors, based on the number of events within the given bin. The pointer indicates the event strength threshold used for the analysis, which corresponds to an interpolated false alarm rate of less than $5 \times 10^{-4} \mathrm{~Hz}$. Note that the signal region data is not included in this calculation. The position of symbols correspond to the center of the bins. 
fix the event strength threshold used in the subsequent analysis.

We chose an event strength threshold with an associated false alarm rate of less than $\simeq 5 \times 10^{-4} \mathrm{~Hz}$, equivalent to less than $\simeq 9 \%$ chance for a false alarm within the $180 \mathrm{sec}$ long signal region.

\section{B. Efficiency determination}

The detection sensitivity of the analysis was determined by simultaneously adding simulated signals of various amplitudes and waveforms to both data streams in the background region and evaluating the efficiency of their detection as a function of the injected amplitude and waveform type.

The waveforms we considered include sine-Gaussians to emulate short narrow-band bursts, Gaussians to emulate short broadband signals, and Dimmelmeier-Font-Müller numerical waveforms [26], as examples of astrophysically motivated signals.

Calibration of the waveforms from strain to ADC counts was performed in the frequency domain, and was done separately for each interferometer. Calibration procedures of the LIGO detectors are described in Ref. [52]. The transformed signals, now in units of counts of raw interferometer noise, were then simply added to the raw data stream.

The amplitudes and the times of the injections were randomly varied. In this way we ensured that each amplitude region sampled the full, representative range of noise variations and that we had no systematic effects, for example, due to a regular spacing in time.

To a reasonable approximation the sensitivity of our analysis pipeline can be expressed in terms of the frequency content, the duration and the strength of the gravitational-wave signal. Therefore, it is sufficient to estimate the sensitivity of our search for a representative set of broad and narrow band waveforms, which span the range of frequencies, bandwidth, and duration we wish to search.

We characterize the strength of an arbitrary waveform by its root-sum-square amplitude $\left(h_{\mathrm{RSS}}\right)$, which is defined as [56]:

$$
h_{\mathrm{RSS}}=\sqrt{\int_{-\infty}^{\infty}|h(t)|^{2} d t} .
$$

The above definition of $h_{\text {RSS }}$ includes all frequencies, while the gravitational-wave detectors and search algorithms are only sensitive in a restricted frequency band. In principle, one can analogously define a "band-limited" $h_{\mathrm{RSS}}$, in which only the sensitive frequency band of the analysis is taken into account. Within this paper we choose to adopt the Eq. (6.1) definition of $h_{\mathrm{RSS}}$ for historical reasons.

The extracted sensitivities (see the examples in Figs. 6 and 7) can be used to generalize our measurements and

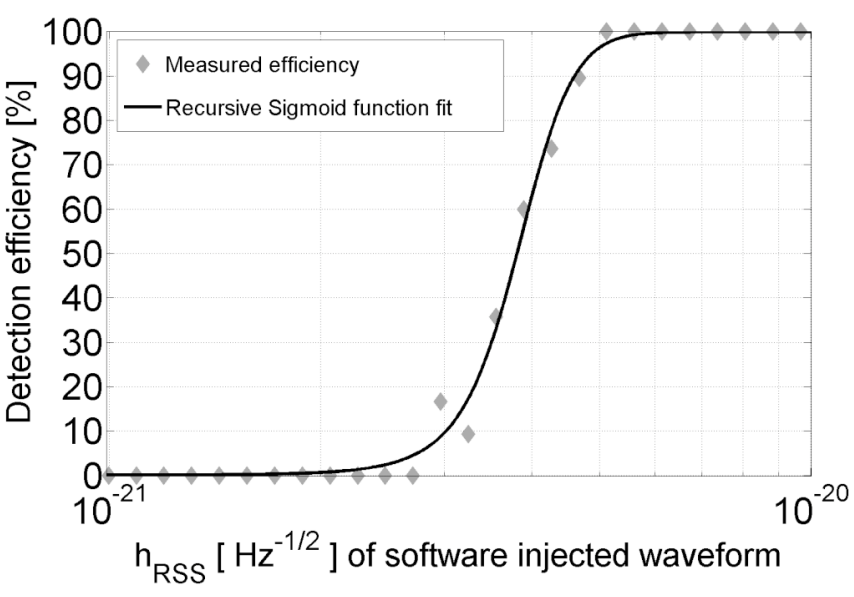

FIG. 6. Efficiency of the detection algorithm for a sample waveform as a function of signal strength $\left(h_{\mathrm{RSS}}\right)$; in this case a sine Gaussian of $f_{0}=250 \mathrm{~Hz}$ and $Q=8.9$. To extract this curve numerous simulated waveforms were embedded in a representative fraction of the background data at random times with randomly varying signal strength. The plot shows the fraction of signals detected as the function of amplitude and a sigmoid function fit. The reconstructed signal onset times were required to fall within $\pm 60 \mathrm{~ms}$ of the true onsets, which also explains why the low $h_{\mathrm{RSS}}$ end of the curve falls near zero. This is a typical plot and in general, the agreement between the measured values, and the fit is better than $\simeq 5 \%$. We relied on the fit to extract our upper limits for an optimally oriented and polarized source. Section VIE below describes the corrections due to nonoptimal source direction and polarization.

estimate the pipeline's sensitivity for other similar bandlimited waveforms.

To assess the sensitivity for relatively narrow band waveforms, we used sine-Gaussian injections of the form:

$$
h(t)=h_{0} \sin \left(\omega_{0} t\right) e^{-t^{2} / 2 \sigma^{2}},
$$

with a central angular frequency of $\omega_{0}=2 \pi f_{0}$, and $Q=$ $\omega_{0} \sigma=2 \pi f_{0} \sigma$. The relation between $h_{0}$ and $h_{\mathrm{RSS}}$ is given as

$$
\begin{aligned}
h_{\mathrm{RSS}}^{\mathrm{SG}} & =h_{0} \sqrt{((\sqrt{\pi} \sigma) / 2)\left(1-e^{-Q^{2}}\right)} \\
& \approx h_{0} \sqrt{Q /\left(4 \sqrt{\pi} f_{0}\right)}\left\{\begin{array}{cc}
1 & Q \gg 1 \\
0.8 & Q \simeq 1
\end{array}\right\} .
\end{aligned}
$$

The injected signals covered the frequency range between 100 and $1850 \mathrm{~Hz}$ with 13 values of $f_{0}$. To test the dependence of the sensitivity on signal duration, we used three values of $Q(4.5,8.9$ and 18) for each frequency (see Table I). Near the most sensitive frequency region, around $\simeq 250 \mathrm{~Hz}$, our gravitational-wave strain sensitivity for optimally polarized bursts was better than $h_{\mathrm{RSS}} \simeq 5 \times$ $10^{-21} \mathrm{~Hz}^{-1 / 2}$. Figure 8 shows the sensitivity for these narrow band waveforms. The symbols mark the simulated event strength $\left(h_{\mathrm{RSS}}\right)$ necessary to achieve $90 \%$ detection efficiency for each waveform. We quote the gravitational- 


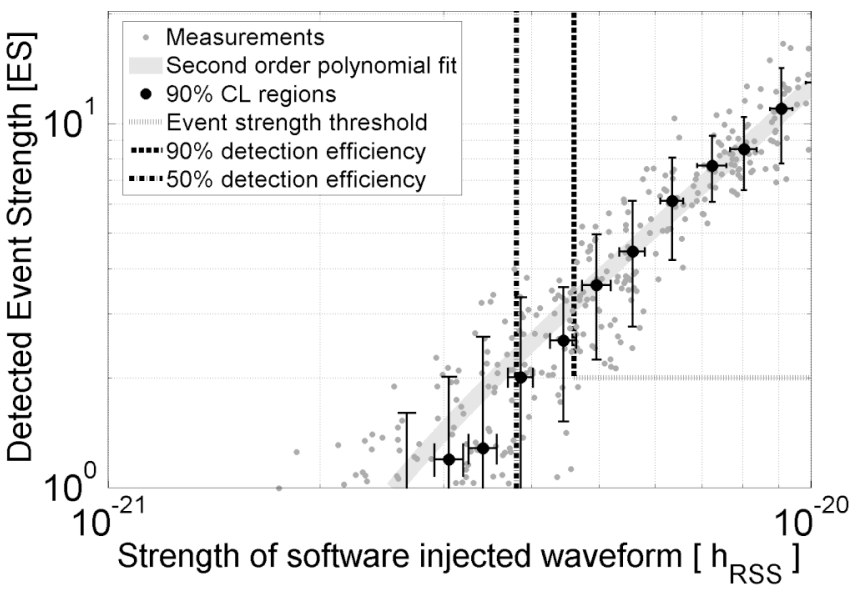

FIG. 7. Detected event strength versus $h_{\text {RSS }}$ of the injected Sine-Gaussian waveform with $f_{0}=250 \mathrm{~Hz}$ and $Q \approx 8.9$. The dots indicate the scatter of the distribution of raw measurements. The gray band shows the quadratic polynomial fit, which allows us to convert the strength of an observed event into the equivalent $h_{\mathrm{RSS}}$ value and determine the associated $90 \%$ C.L. error bars. The markers with error bars represent the $90 \%$ C.L. regions for subsets of the data. For each marker, $90 \%$ of the measurements used were within the horizontal error bars and $90 \%$ of the detected event strengths values fell within the vertical error bars. The vertical dash-dotted line represents the $50 \%$ detection efficiency associated with the waveform type and the chosen detection threshold (horizontal dotted line). As expected, the crossing of the threshold and the $50 \%$ efficiency lines agree well with the fit and the center of the corresponding marker. The vertical dashed line represents the boundary of the region where we have better than $90 \%$ detection efficiency. The "corner" defined by the event strength threshold and the $90 \%$ detection efficiency boundary (dashed lines) agrees well with the curve outlined by the lower end of the vertical error bars of the markers. All events in the upper right corner of the plot (above and beyond the dashed lines) are detectable with high confidence. This plot is typical for different waveforms considered in the analysis.

wave signal strength associated with the $90 \%$ detection efficiency, as this can be related to the upper limits on the gravitational-wave strength associated with the source. Figure 8 also illustrates the insensitivity of the detection efficiency to the $Q$ of the sine-Gaussian waveforms with the same central frequency, as these reach their $90 \%$ efficiency levels at similar gravitational-wave strengths, even though their $Q$ differ by a factor of $\simeq 4$; for a given $h_{\mathrm{RSS}}$, a longer signal (higher $Q$ ) would of course, have a smaller $h_{\text {PEAK}}$. This strength is frequency dependent, naturally following the frequency dependence of the detector sensitivities, which are also indicated in Fig. 8.

Table II shows a similar set of efficiencies estimated using broadband simulated signals. We used two types of broadband waveforms, sine Gaussians with unity quality factor and Gaussians. Both are short bursts, however, the Gaussians are even functions while the sine Gaussians are odd, leading to different peak amplitudes with the same
TABLE I. $h_{\mathrm{RSS}}\left[\mathrm{Hz}^{-1 / 2}\right]$ for $90 \%$ detection efficiency for sineGaussians (SG) waveforms at various frequencies $\left(f_{0}\right)$ and $Q$ [see Eq. (6.2)]. The quoted values are the results of simulations and are subject to a total of $\simeq 15 \%$ statistical and systematic errors, which are taken into account when quoting the $U L_{h_{\mathrm{RSS}}}^{90 \% \mathrm{C} . \mathrm{L} \text {. }}$ values. Note that at the low and at the high frequency end, the low $Q$ waveforms have significant power outside of the analysis frequency band.

\begin{tabular}{|c|c|c|c|c|c|}
\hline Waveform & $\sigma[\mathrm{ms}]$ & $Q$ & $f_{0}[\mathrm{~Hz}]$ & $h_{\mathrm{RSS}}^{90 \%}\left[\mathrm{~Hz}^{-1 / 2}\right]$ & $U L_{h_{\mathrm{RSS}}}^{90 \% \mathrm{C} . \mathrm{L}}\left[\mathrm{Hz}^{-1 / 2}\right]$ \\
\hline SG & 7.2 & 4.5 & 100 & $17 \times 10^{-21}$ & $20 \times 10^{-21}$ \\
\hline SG & 2.9 & 4.5 & 250 & $4.8 \times 10^{-21}$ & $5.6 \times 10^{-21}$ \\
\hline SG & 2 & 4.5 & 361 & $5.8 \times 10^{-21}$ & $6.7 \times 10^{-21}$ \\
\hline SG & 1.6 & 4.5 & 458 & $7.0 \times 10^{-21}$ & $8.0 \times 10^{-21}$ \\
\hline SG & 1.3 & 4.5 & 554 & $7.9 \times 10^{-21}$ & $9.1 \times 10^{-21}$ \\
\hline SG & 1 & 4.5 & 702 & $10 \times 10^{-21}$ & $11 \times 10^{-21}$ \\
\hline SG & 0.84 & 4.5 & 850 & $12 \times 10^{-21}$ & $14 \times 10^{-21}$ \\
\hline SG & 0.72 & 4.5 & 1000 & $15 \times 10^{-21}$ & $17 \times 10^{-21}$ \\
\hline SG & 0.53 & 4.5 & 1361 & $27 \times 10^{-21}$ & $31 \times 10^{-21}$ \\
\hline SG & 0.49 & 4.5 & 1458 & $30 \times 10^{-21}$ & $34 \times 10^{-21}$ \\
\hline SG & 0.46 & 4.5 & 1554 & $37 \times 10^{-21}$ & $43 \times 10^{-21}$ \\
\hline SG & 0.42 & 4.5 & 1702 & $43 \times 10^{-21}$ & $50 \times 10^{-21}$ \\
\hline SG & 0.39 & 4.5 & 1850 & $50 \times 10^{-21}$ & $58 \times 10^{-21}$ \\
\hline SG & 14 & 8.9 & 100 & $18 \times 10^{-21}$ & $21 \times 10^{-21}$ \\
\hline SG & 5.7 & 8.9 & 250 & $4.6 \times 10^{-21}$ & $5.3 \times 10^{-21}$ \\
\hline SG & 3.9 & 8.9 & 361 & $6.0 \times 10^{-21}$ & $6.9 \times 10^{-21}$ \\
\hline SG & 3.1 & 8.9 & 458 & $7.1 \times 10^{-21}$ & $8.1 \times 10^{-21}$ \\
\hline SG & 2.6 & 8.9 & 554 & $7.3 \times 10^{-21}$ & $8.4 \times 10^{-21}$ \\
\hline SG & 2 & 8.9 & 702 & $8.9 \times 10^{-21}$ & $10 \times 10^{-21}$ \\
\hline SG & 1.7 & 8.9 & 850 & $10 \times 10^{-21}$ & $12 \times 10^{-21}$ \\
\hline SG & 1.4 & 8.9 & 1000 & $13 \times 10^{-21}$ & $15 \times 10^{-21}$ \\
\hline SG & 1 & 8.9 & 1361 & $20 \times 10^{-21}$ & $23 \times 10^{-21}$ \\
\hline SG & 0.97 & 8.9 & 1458 & $23 \times 10^{-21}$ & $27 \times 10^{-21}$ \\
\hline SG & 0.91 & 8.9 & 1554 & $26 \times 10^{-21}$ & $30 \times 10^{-21}$ \\
\hline SG & 0.83 & 8.9 & 1702 & $32 \times 10^{-21}$ & $37 \times 10^{-21}$ \\
\hline SG & 0.77 & 8.9 & 1850 & $38 \times 10^{-21}$ & $44 \times 10^{-21}$ \\
\hline SG & 29 & 18 & 100 & $23 \times 10^{-21}$ & $26 \times 10^{-21}$ \\
\hline SG & 11 & 18 & 250 & $5.0 \times 10^{-21}$ & $5.7 \times 10^{-21}$ \\
\hline SG & 7.9 & 18 & 361 & $6.4 \times 10^{-21}$ & $7.4 \times 10^{-21}$ \\
\hline SG & 6.3 & 18 & 458 & $7.9 \times 10^{-21}$ & $9.1 \times 10^{-21}$ \\
\hline SG & 5.2 & 18 & 554 & $7.7 \times 10^{-21}$ & $8.9 \times 10^{-21}$ \\
\hline SG & 4.1 & 18 & 702 & $9.8 \times 10^{-21}$ & $11 \times 10^{-21}$ \\
\hline SG & 3.4 & 18 & 850 & $10 \times 10^{-21}$ & $12 \times 10^{-21}$ \\
\hline SG & 2.9 & 18 & 1000 & $12 \times 10^{-21}$ & $14 \times 10^{-21}$ \\
\hline SG & 2.1 & 18 & 1361 & $19 \times 10^{-21}$ & $21 \times 10^{-21}$ \\
\hline SG & 2 & 18 & 1458 & $21 \times 10^{-21}$ & $24 \times 10^{-21}$ \\
\hline SG & 1.8 & 18 & 1554 & $22 \times 10^{-21}$ & $25 \times 10^{-21}$ \\
\hline SG & 1.7 & 18 & 1702 & $29 \times 10^{-21}$ & $33 \times 10^{-21}$ \\
\hline SG & 1.5 & 18 & 1850 & $34 \times 10^{-21}$ & $39 \times 10^{-21}$ \\
\hline
\end{tabular}

$h_{\mathrm{RSS}}$ value. Gaussians were parametrized as

$$
h(t)=h_{0} e^{-t^{2} / 2 \sigma^{2}} .
$$

The relationship between $h_{0}$ and $h_{\mathrm{RSS}}$ for a Gaussian is

$$
h_{\mathrm{RSS}}^{\mathrm{GA}}=h_{0} \sqrt{\sqrt{\pi} \sigma} .
$$




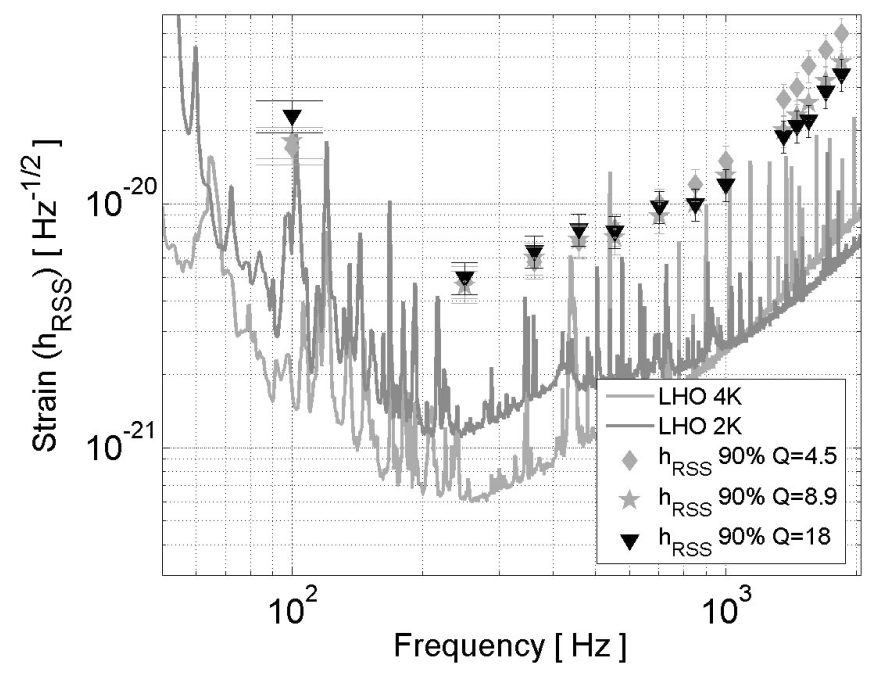

FIG. 8. Sensitivity of the detection algorithm for detecting sine-Gaussian waveforms versus characteristic frequency. The plot shows the strength necessary for $90 \%$ detection efficiency. The gray spectra illustrate the sensitivity of the $2 \mathrm{~K}$ and $4 \mathrm{~K}$ Hanford detectors during the time surrounding the GRB030329 trigger. The error bars reflect a total $15 \%$ error.

TABLE II. As in Table I, $h_{\mathrm{RSS}}\left[\mathrm{Hz}^{-1 / 2}\right]$ for $90 \%$ detection efficiency for Gaussian (GA) waveforms of various durations $(\sigma)$ [see Eq. (6.4)] and for sine-Gaussian (SG) waveforms at various frequencies $\left(f_{0}\right)$ and $Q=1$ [see Eq. (6.2)]. Note that these broadband waveforms have significant power outside of the analysis frequency band.

\begin{tabular}{lccccc}
\hline \hline Waveform & $\sigma[\mathrm{ms}]$ & $\mathrm{Q}$ & $f_{0}[\mathrm{~Hz}]$ & $h_{\mathrm{RSS}}^{90 \%}\left[\mathrm{~Hz}^{-1 / 2}\right]$ & $U L_{h_{\mathrm{RSS}}}^{90 \% \mathrm{~L} .}\left[\mathrm{Hz}^{-1 / 2}\right]$ \\
\hline SG & 1.6 & 1 & 100 & $10 \times 10^{-21}$ & $12 \times 10^{-21}$ \\
SG & 0.64 & 1 & 250 & $6.5 \times 10^{-21}$ & $7.4 \times 10^{-21}$ \\
SG & 0.44 & 1 & 361 & $8.4 \times 10^{-21}$ & $9.7 \times 10^{-21}$ \\
SG & 0.35 & 1 & 458 & $10 \times 10^{-21}$ & $12 \times 10^{-21}$ \\
SG & 0.29 & 1 & 554 & $13 \times 10^{-21}$ & $14 \times 10^{-21}$ \\
SG & 0.23 & 1 & 702 & $18 \times 10^{-21}$ & $20 \times 10^{-21}$ \\
SG & 0.19 & 1 & 850 & $23 \times 10^{-21}$ & $26 \times 10^{-21}$ \\
SG & 0.16 & 1 & 1000 & $26 \times 10^{-21}$ & $30 \times 10^{-21}$ \\
SG & 0.12 & 1 & 1361 & $39 \times 10^{-21}$ & $45 \times 10^{-21}$ \\
SG & 0.11 & 1 & 1458 & $44 \times 10^{-21}$ & $51 \times 10^{-21}$ \\
SG & 0.1 & 1 & 1554 & $46 \times 10^{-21}$ & $52 \times 10^{-21}$ \\
SG & 0.094 & 1 & 1702 & $55 \times 10^{-21}$ & $63 \times 10^{-21}$ \\
SG & 0.086 & 1 & 1850 & $61 \times 10^{-21}$ & $70 \times 10^{-21}$ \\
\hline GA & 0.5 & & & $8.3 \times 10^{-21}$ & $9.6 \times 10^{-21}$ \\
GA & 0.75 & & & $9.6 \times 10^{-21}$ & $1.1 \times 10^{-20}$ \\
GA & 1 & & & $1.3 \times 10^{-20}$ & $1.5 \times 10^{-20}$ \\
GA & 2 & & & $3.3 \times 10^{-20}$ & $3.8 \times 10^{-20}$ \\
GA & 3 & & & $8.2 \times 10^{-20}$ & $9.5 \times 10^{-20}$ \\
GA & 4 & & & $1.9 \times 10^{-19}$ & $2.2 \times 10^{-19}$ \\
GA & 5.5 & & $8.5 \times 10^{-19}$ & $9.8 \times 10^{-19}$ \\
GA & 8 & & & $1.3 \times 10^{-17}$ & $1.5 \times 10^{-17}$ \\
GA & 10 & & & $1.0 \times 10^{-16}$ & $1.2 \times 10^{-16}$ \\
& & & & & \\
\hline & & & & \\
\hline
\end{tabular}

The estimated sensitivities indicate that the $90 \%$ detection efficiency limits for short bursts are similar to those obtained for the narrow band waveforms when one takes into account that only part of the power of the broadband waveforms is confined to the analysis frequency band. Longer Gaussian bursts are more difficult to detect, as their spectrum has a significant low frequency component, outside the sensitive band of our analysis.

We have also estimated our efficiency for a set of astrophysically motivated burst waveforms [26] (see Table III). These simulated waveforms are not expected to be necessarily associated with GRBs, rather these results are presented here to further illustrate the waveform independence of the analysis.

\section{Signal region}

The analysis of the signal region (Fig. 9) yielded only events well below the predetermined event strength threshold ( $\lesssim 60 \%$ of threshold). Since we had no candidate event, we placed an upper limit on the detected strength of gravitational waves associated to GRB030329. Our fixed

TABLE III. As in Table I, $h_{\mathrm{RSS}}\left[\mathrm{Hz}^{-1 / 2}\right]$ for $90 \%$ detection efficiency for astrophysically motivated waveforms. These waveforms are described in detail in Ref. [26]. Note that most of these waveforms have significant power outside of the analysis frequency band.

\begin{tabular}{lccc}
\hline \hline Simulation & Waveform & $h_{\mathrm{RSS}}^{90 \%}\left[\mathrm{~Hz}^{-1 / 2}\right]$ & $U L_{h_{\mathrm{RSS}}}^{90 \% \mathrm{C} \cdot\left[\mathrm{Hz}^{-1 / 2}\right]}$ \\
\hline DFM & A1B1G1 & $12 \times 10^{-21}$ & $14 \times 10^{-21}$ \\
DFM & A1B2G1 & $13 \times 10^{-21}$ & $15 \times 10^{-21}$ \\
DFM & A1B3G1 & $12 \times 10^{-21}$ & $14 \times 10^{-21}$ \\
DFM & A1B3G2 & $12 \times 10^{-21}$ & $14 \times 10^{-21}$ \\
DFM & A1B3G3 & $12 \times 10^{-21}$ & $14 \times 10^{-21}$ \\
DFM & A1B3G5 & $34 \times 10^{-21}$ & $39 \times 10^{-21}$ \\
DFM & A2B4G1 & $24 \times 10^{-21}$ & $27 \times 10^{-21}$ \\
DFM & A3B1G1 & $19 \times 10^{-21}$ & $21 \times 10^{-21}$ \\
DFM & A3B2G1 & $20 \times 10^{-21}$ & $23 \times 10^{-21}$ \\
DFM & A3B2G2 & $15 \times 10^{-21}$ & $17 \times 10^{-21}$ \\
DFM & A3B2G4 & $14 \times 10^{-21}$ & $16 \times 10^{-21}$ \\
DFM & A3B3G1 & $28 \times 10^{-21}$ & $33 \times 10^{-21}$ \\
DFM & A3B3G2 & $17 \times 10^{-21}$ & $20 \times 10^{-21}$ \\
DFM & A3B3G3 & $12 \times 10^{-21}$ & $14 \times 10^{-21}$ \\
DFM & A3B3G5 & $30 \times 10^{-21}$ & $34 \times 10^{-21}$ \\
DFM & A3B4G2 & $23 \times 10^{-21}$ & $27 \times 10^{-21}$ \\
DFM & A3B5G4 & $26 \times 10^{-21}$ & $29 \times 10^{-21}$ \\
DFM & A4B1G1 & $38 \times 10^{-21}$ & $44 \times 10^{-21}$ \\
DFM & A4B1G2 & $32 \times 10^{-21}$ & $36 \times 10^{-21}$ \\
DFM & A4B2G2 & $42 \times 10^{-21}$ & $48 \times 10^{-21}$ \\
DFM & A4B2G3 & $39 \times 10^{-21}$ & $45 \times 10^{-21}$ \\
DFM & A4B4G4 & $17 \times 10^{-21}$ & $19 \times 10^{-21}$ \\
DFM & A4B4G5 & $12 \times 10^{-21}$ & $13 \times 10^{-21}$ \\
DFM & A4B5G4 & $21 \times 10^{-21}$ & $25 \times 10^{-21}$ \\
DFM & A4B5G5 & $19 \times 10^{-21}$ & $22 \times 10^{-21}$ \\
\hline \hline
\end{tabular}




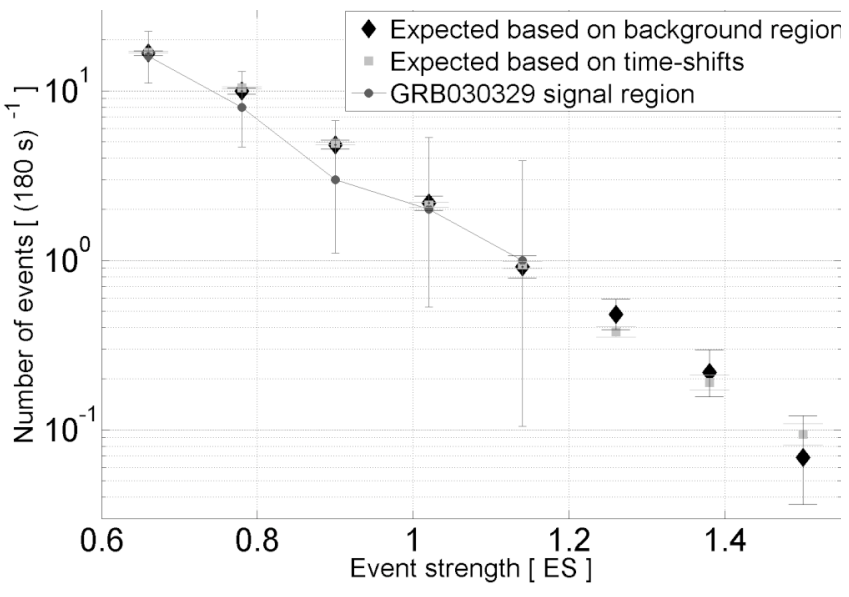

FIG. 9. Number of events versus event strength in the signal region (circle). The diamonds show the expected distribution based on the background region. The squares mark the expected distribution based on nonphysical time shifts (ranging from 2 to $9 \mathrm{sec}$ ) between the $\mathrm{H} 1$ and $\mathrm{H} 2$ data streams in the background region. The error bars reflect $90 \%$ C.L. Poisson errors. The position of the symbols correspond to the center of the bins.

false alarm rate permitted the results of simulations to be used directly in setting upper limits.

The upper limits on $h_{\mathrm{RSS}}$ for narrow band waveforms are given in Table I. Tables II and III show the upper limits for the broadband simulations and astrophysically motivated waveforms, respectively.

\section{Errors}

The analysis method, the procedures used to determine the efficiencies, and the nonstationary nature of the data, all contribute to the uncertainty associated with the results.

The efficiency (versus $h_{\mathrm{RSS}}$ ) values have an estimated $\simeq 11 \%$ uncertainty due to our limited knowledge of the calibrated response of our detectors. This estimate also accounts for the slight difference in calibrated response between the signal region and background data used for the simulations.

An additional uncertainty arises from the nonstationarity of the data. The results of the simulations exhibit a slight dependence on the choice of the actual data segments ("base" data) used for the injections. This dependence was characterized via simulations using numerous different subsegments of the background data. We repeated the full efficiency estimation process several times for the same waveform, while injecting into various base data stretches. The variation in the measured upper limits indicated $\simeq 10 \%$ uncertainty due to the dependency of our upper limits on the base data. This uncertainty shall also account for the statistical error due to the finite number of simulations used.

We characterized the detection efficiencies for each waveform considered via fits of sigmoid functions (see, for example, Fig. 6). The fits agree well with the data, but small differences are occasionally observed in the $\gtrsim 90 \%$ efficiency region. We estimate that using these fits can underestimate the $90 \%$ limits by $\lesssim 5 \%$.

The uncertainties listed above are taken into account by specifying a total $15 \%$ uncertainty for each measurement in Fig. 8 and in all tables.

The false alarm rate associated with the results was also measured. The false alarm rate limit is based on the measurement with zero lag data plus the $90 \%$ confidence Poisson error bars. We have checked the assumption of Poisson background statistics by examining the time intervals between consecutive triggers and the variance in trigger counts for varying ES thresholds when the background sample is divided into 50 equal-length intervals. Good agreement with the Poisson expectation is observed. This choice provides a conservative estimate of our associated $\left(\simeq 5 \times 10^{-4} \mathrm{~Hz}\right.$ ) false alarm rate.

\section{E. Astrophysical interpretation}

GRB030329 has a well-determined redshift, therefore we can relate our observed limits on strain to a measure of the total gravitational-wave energy emission. For a strain $h(t)$ at distance $D_{L}$ from a source of gravitational radiation, the associated power is proportional to $\dot{h}^{2}(\dot{h}=d h / d t)$, though the proportionality constant will depend on the (unknown) emission pattern of the source and the antenna pattern of the detector (for the known source position, but unknown polarization angle).

In general, it is not possible to relate our upper limit on the strain from a particular waveform to a limit on the energy radiated by the source, without assuming a model. Sources that radiate energy $E_{\mathrm{GW}}$ might produce an arbitrarily small signal $h(t)$ in the detector, e.g., if the dynamics in the source were purely axisymmetric with the detector located on the axis. Nevertheless, we can associate a strain $h(t)$ in the detector with some minimum amount of gravitational-wave energy radiated by the source by choosing an "optimistic" emission pattern, thereby obtaining a measure of the minimum amount of energy that would need to be radiated in order to obtain a detectable signal. We will show that the progenitor of GRB030329 is not expected to have produced a detectable signal.

We are interested in a "plausible case scenario" of gravitational-wave emission in order to obtain the minimum (plausible) amount of gravitational-wave energy radiated that could be associated with a detector signal $h(t)$. We do not expect the gravitational waves to be strongly beamed, and we expect that we are observing the GRB progenitor along some preferred axis. We take a model best case scenario to be that of gravitational-wave emission from a triaxial ellipsoid rotating about the same axis as the GRB (i.e., the direction to the Earth). If we assume quadrupolar gravitational-wave emission, the plusand cross-polarization waveforms, emitted at a polar angle 
$\theta$ from the axis of rotation to be

$$
\begin{gathered}
h_{+}=\frac{1}{2}\left(1+\cos ^{2} \theta\right) h_{+, 0} \\
h_{\times}=\cos \theta h_{\times, 0}
\end{gathered}
$$

where $h_{+, 0}$ and $h_{\times, 0}$ are two orthogonal waveforms (e.g., a sine Gaussian and a cosine Gaussian), each containing the same amount of radiative power. That is, we assume that the same amount of gravitational-wave energy is carried in the two polarizations and that they are orthogonal:

$$
\int_{-\infty}^{\infty} \dot{h}_{+, 0}^{2} d t=\int_{-\infty}^{\infty} \dot{h}_{\times, 0}^{2} d t \quad \text { and } \quad \int_{-\infty}^{\infty} \dot{h}_{+, 0} \dot{h}_{\times, 0} d t=0 .
$$

Thus, we would expect that the gravitational waves travelling along the rotational axis (toward the Earth) would be circularly polarized, and that the detector would receive the signal

$$
h=F_{+} h_{+, 0}+F_{\times} h_{\times, 0}
$$

where $F_{+}$and $F_{\times}$represent the detector responses to the polarization components $h_{+, 0}$ and $h_{\times, 0}$ [57], and depend on the position of the source in the sky and on a polarization angle. The radiated energy from such a system is calculated to be

$$
\begin{aligned}
E_{\mathrm{GW}} & =\frac{c^{3}}{16 \pi G} \int d A \int_{-\infty}^{\infty}\left(\dot{h}_{+}^{2}+\dot{h}_{\times}^{2}\right) d t \\
& =\frac{c^{3}}{5 G} \frac{D_{L}^{2}}{\eta^{2}} \int_{-\infty}^{\infty} \dot{h}^{2} d t,
\end{aligned}
$$

where $\eta^{2}=F_{+}^{2}+F_{\times}^{2}$ (which depends only on the position of the source on the sky) and where we are integrating over a spherical shell around the source with radius $D_{L}$ (the distance to the Earth). Alternatively, using Parseval's identity, we have

$$
E_{\mathrm{GW}}=\frac{8 \pi^{2} c^{3}}{5 G} \frac{D_{L}^{2}}{\eta^{2}} \int_{0}^{\infty}|f \tilde{h}|^{2} d f
$$

where

$$
\tilde{h}(f)=\int_{-\infty}^{\infty} h(t) e^{-2 \pi i f t} d t .
$$

Whereas optimal orientation gives $\eta=1$ for a source at zenith, the position of GRB030329 was far from optimal. The angle with respect to zenith was $68^{\circ}$ and the azimuth with respect to the $x$ arm was $45^{\circ}$, which yields $\eta=0.37$.

We now relate $E_{\mathrm{GW}}$ to the strain upper limits using the specific waveforms used in the analysis. For a Gaussian waveform [see Eq. (6.4)]:

$$
E_{\mathrm{GW}}=\left(\frac{\sqrt{\pi} c^{3}}{10 G}\right)\left(\frac{D_{L}^{2} h_{0}^{2}}{\sigma}\right)
$$

and for a sine-Gaussian waveform [see Eq. (6.2)]:

$$
E_{\mathrm{GW}}=\left(\frac{\sqrt{\pi} c^{3}}{20 G}\right)\left(\frac{D_{L}^{2} h_{0}^{2}}{\sigma}\right)\left(1+2 Q^{2}-e^{-Q^{2}}\right)
$$

where $Q=\omega_{0} \sigma=2 \pi f_{0} \sigma$. The relation between $h_{0}$ and $h_{\mathrm{RSS}}$ is given in Eqs. (6.3) and (6.5).

We can relate the observed limit on $h_{\mathrm{RSS}}$ to an equivalent mass $M_{\mathrm{EQ}}$ which is converted to gravitational radiation with $100 \%$ efficiency, $E_{\mathrm{GW}}=M_{\mathrm{EQ}} c^{2}$, at a luminosity distance $D_{L} \approx 800 \mathrm{Mpc}$. For sine-Gaussian waveforms with $f_{0}=250 \mathrm{~Hz}$ and $Q=8.9, M_{\mathrm{EQ}}=1.9 \times 10^{4} \eta^{-2} M_{\odot}$. For Gaussian waveforms with $\sigma=1 \mathrm{~ms}, M_{\mathrm{EQ}}=3.1 \times$ $10^{4} \eta^{-2} M_{\odot}$. However, we would not expect that the gravitational-wave luminosity of the source could exceed $\simeq c^{5} / G=2 \times 10^{5} M_{\odot} c^{2}$ per second [58], so we would not expect an energy in gravitational waves much more than $\simeq 2 \times 10^{3} M_{\odot} c^{2}$ in the $\simeq 10 \mathrm{~ms}$ sine-Gaussian waveform, or an energy of much more than $\simeq 3 \times 10^{4} M_{\odot} c^{2}$ in the maximum duration $(150 \mathrm{~ms})$ of the search; far below the limits on $M_{\mathrm{EQ}} c^{2}$ that we find in this analysis. Present theoretical expectations on the gravitational-wave energy emitted range from $10^{-6} M_{\odot} c^{2}-10^{-4} M_{\odot} c^{2}$ to $10^{-1} M_{\odot} c^{2}-M_{\odot} c^{2}$ for some of the most optimistic models [see e.g. [5,55,59,60]]. Nonetheless, these scalings indicate how we can probe well below these energetic limits with future analyses. For example, assuming similar detector performance for an optimally oriented trigger like GRB980425 $\left(D_{L} \approx 35 \mathrm{Mpc}\right)$ the limit on the equivalent mass would be $M_{\mathrm{EQ}} \approx 60 M_{\odot}$ for the Gaussian waveforms mentioned above with $\sigma=1 \mathrm{~ms}$.

\section{SUMMARY}

\section{A. Comparison with previous searches for gravitational waves from GRBs}

Our result is comparable to the best published results searching for association between gravitational waves and GRBs [61], however these studies differ in their most sensitive frequency.

Tricarico et al. [62] used a single resonant mass detector, AURIGA [63], to look for an excess in coincidences between the arrival times of GRBs in the BATSE 4B catalog. They used two different methods. They searched for events identified above a certain threshold in the gravitational-wave data, and also attempted to establish a statistical association between GRBs and gravitational waves. No significant excess was found with the former method. The latter used a variant of the correlation based Finn-Mohanty-Romano (FMR) method [64]. However, instead of using the cross correlation of two detectors, as proposed in the FMR method, only the variance of the single detector output was used. A sample of variances from times when there were no GRBs was compared with a corresponding sample from data that spanned the arrival times of the GRBs. An upper limit on the source-averaged gravitational-wave signal root mean square value of $1.5 \times$ $10^{-18}$ was found using $120 \mathrm{GRBs}$. This limit applies at the 
AURIGA resonant frequencies of 913 and $931 \mathrm{~Hz}$, which are very far from the most sensitive frequency of the LIGO detectors $(\simeq 250 \mathrm{~Hz})$. This work [62] was later extended [65], which led to an improved upper limit.

The data analysis method employed in Modestino and Moleti [66] is another variant of the FMR method. Instead of constructing off-source samples from data segments that are far removed from the GRB trigger, the off-source samples are constructed by introducing nonzero time shifts between the two detector data streams and computing their cross correlation. For narrowband resonant mass detectors, the directional information of a GRB cannot be exploited to discriminate against incorrect relative timing since the signal in the output of the detector is spread out by the detector response over time scales much larger than the light travel time between the detectors.

Astone et al. [67,68] report on a search for a statistical association between GRBs and gravitational waves using data from the resonant mass detectors EXPLORER [69] and NAUTILUS [70]. They report a Bayesian upper limit on gravitational-wave signal amplitudes of $1.2 \times 10^{-18}$, at 95\% probability, when the maximum delay between the GRB and gravitational wave is kept at $400 \mathrm{sec}$. The upper limit improves to $6.5 \times 10^{-19}$ when the maximum delay is reduced to $5 \mathrm{sec}$. However, the absence of directional and/ or distance information for most of these GRBs precluded accounting for source variations; the gravitational-wave signal amplitude was assumed to be the same for all of the GRBs.

Astone et al. [71] report on the operation of the resonant mass detectors EXPLORER during the closest ever gamma ray burst (GRB980425) with known redshift and direction. At the time of the burst, EXPLORER was taking data with close to optimal orientation. GRB980425 was $\simeq 23$ times closer to Earth than GRB030329 giving a $\simeq 520$ increase in energy sensitivity. Based on their sensitivity and the loudest event within \pm 5 minutes of the GRB980425 trigger the authors quote a limit of $\simeq 1600 M_{\odot}$ for a simple model assuming isotropic gravitational-wave emission.

Recently, Astone et al. [61] executed a search aiming to detect a statistical association between the GRBs detected by the satellite experiments BATSE and BeppoSAX, and the EXPLORER and NAUTILUS gravitational-wave detectors. No association was uncovered. Their upper limit is the lowest published result, which is based on bar-detector gravitational-wave data.

\section{B. Conclusion}

We have executed a cross-correlation-based search for possible gravitational-wave signatures around the GRB030329 trigger, which occurred during the second science run of the LIGO detectors. We analyzed a $180 \mathrm{sec}$ signal region around the GRB and $4.5 \mathrm{~h}$ of background data, surrounding the signal region, corresponding to a single coincident lock stretch. These data were sufficient to characterize the background, scan the signal region and estimate our efficiency. We used the same procedure, based on cross correlation, for each of these studies. We evaluated the sensitivity of the search to a large number of broad and narrow band waveforms.

We observed no candidates with gravitational-wave signal strength larger than a predetermined threshold, therefore we set upper limits on the associated gravitationalwave strength at the detectors. The present analysis covers the most sensitive frequency range of the Hanford detectors, approximately from 80 to $2048 \mathrm{~Hz}$. The frequencydependent sensitivity of our search was $h_{\mathrm{RSS}} \simeq$ $\mathrm{O}\left(6 \times 10^{-21}\right) \mathrm{Hz}^{-1 / 2}$.

The prospect for future searches is promising, as the sensitivity of the instruments improves with further commissioning.

Once operating at target sensitivity, the detectors will be more sensitive to strain than they were during S2 by factors of 10-100, depending on frequency (see Fig. 2.). This implies an improvement of a factor of $\sim 1000$ in sensitivity to $E_{\mathrm{GW}}$, since $E_{\mathrm{GW}}$ scales like $\sim h_{\mathrm{RSS}}^{2}$ [see, for example, Eq. (6.13)].

Detection of GRBs with measured redshifts significantly smaller than GRB030329's is certainly possible. GRB030329's electromagnetic brightness was due to a favorable combination of distance and our position in its beam. One year of observation will incorporate hundreds of GRBs with LIGO data coverage and some of these GRBs, even though fainter [72-74] than GRB030329, could be significantly closer, as was $1998 \mathrm{bw}$. We can also hope for sources with more optimal direction and coincidence between three or four observing interferometers.

\section{ACKNOWLEDGMENTS}

The authors gratefully acknowledge the support of the United States National Science Foundation for the construction and operation of the LIGO Laboratory and the Particle Physics and Astronomy Research Council of the United Kingdom, the Max-Planck-Society and the State of Niedersachsen/Germany for support of the construction and operation of the GEO600 detector. The authors also gratefully acknowledge the support of the research by these agencies and by the Australian Research Council, the Natural Sciences and Engineering Research Council of Canada, the Council of Scientific and Industrial Research of India, the Department of Science and Technology of India, the Spanish Ministerio de Ciencia y Tecnologia, the John Simon Guggenheim Foundation, the Leverhulme Trust, the David and Lucile Packard Foundation, the Research Corporation, and the Alfred P. Sloan Foundation. We are grateful to Scott Barthelmy and the GCN network and Kevin Hurley and the IPN network for providing us with near real time GRB triggers and to the Ulysses, Konus, SAX, and HETE experiments, who detect 
and generate the events distributed by GCN and IPN. This research has made use of data obtained from the HETE science team via the website in Ref. [75]. HETE is an international mission of the NASA Explorer program, run by the Massachusetts Institute of Technology.
[1] C. Kouveliotou et al., Astrophys. J. Lett. 413, L101 (1993).

[2] B. Zhang and P. Mészáros, Int. J. Mod. Phys. A 19, 2385 (2004).

[3] C. L. Fryer, S. E. Woosley, and A. Heger, Astrophys. J. 550, 372 (2001).

[4] M. B. Davies et al., Astrophys. J. 579, L63 (2002).

[5] M. H. van Putten et al., Phys. Rev. D 69, 044007 (2004).

[6] D. Q. Lamb et al., New Astron. Rev. 48, 423 (2004).

[7] P. A. Price et al., Nature (London) 423, 844 (2003).

[8] T. Matheson et al., Astrophys. J. 599, 394 (2003).

[9] V. V. Sokolov et al., astro-ph/0312359.

[10] S. R. Kulkarni et al., Nature (London) 395, 663 (1998).

[11] K. Iwamoto et al., Nature (London) 395, 672 (1998).

[12] T. J. Galama et al., Nature (London) 395, 670 (1998).

[13] D. A. Frail et al., Astrophys. J. Lett. 562, L55 (2001).

[14] M.H. P. M. van Putten and T. Regimbau, Astrophys. J. Lett. 593, L15 (2003).

[15] K. Belczynski, T. Bulik, and B. Rudak, Astrophys. J. Lett. 608, L45 (2004).

[16] É.É. Flanagan and S. A. Hughes, Phys. Rev. D 57, 4535 (1998).

[17] J. D. E. Creighton, Phys. Rev. D 60, 022001 (1999).

[18] C. W. Helstrom, Statistical Theory of Signal Detection (Pergamon Press, London, 1968), 2nd ed.

[19] B. Abbott et al., Phys. Rev. D 69, 122001 (2004).

[20] E. Müller et al., Astrophys. J. 603, 221 (2004).

[21] C. L. Fryer and M. S. Warren, Astrophys. J. 601, 391 (2004).

[22] C. D. Ott et al., Astrophys. J. 600, 834 (2004).

[23] A. I. MacFadyen and S. E. Woosley, Astrophys. J. 524, 262 (1999).

[24] A. Heger et al., Astrophys. J. 591, 288 (2003).

[25] A. Burrows and J. Hayes, Phys. Rev. Lett. 76, 352 (1996).

[26] H. Dimmelmeier, J.A. Font, and E. Müller, Astron. Astrophys. 393, 523 (2002).

[27] C. L. Fryer, D. E. Holz, and S. A. Hughes, Astrophys. J. 565, 430 (2002).

[28] T. Zwerger and E. Müller, Astron. Astrophys. 320, 209 (1997).

[29] M. H. P. M. van Putten, Astrophys. J. 583, 374 (2003).

[30] J. C. B. Papaloizou and J. E. Pringle, Mon. Not. R. Astron. Soc. 208, 721 (1984).

[31] R. Vanderspek et al., GRB Circular Network 1997 (2003); also see http://space.mit.edu/HETE/Bursts/GCN/ 1997.gcn

[32] G. R. Ricker, R. K. Vanderspek, and HETE Science Team, American Astronomical Society Meeting 203 (2003); also see http://www.aas.org/publications/baas/v35n5/aas203/ 1192.htm

[33] R. Vanderspek et al., Astrophys. J. 617, 1251 (2004).
[34] S. Golenetskii et al., GRB Circular Network 2026 (2003); also see http://space.mit.edu/HETE/Bursts/GCN/ 2026.gcn

[35] http://space.mit.edu/HETE/Bursts/GRB030329

[36] Y. M. Lipkin et al., Astrophys. J. 606, 381 (2004).

[37] H. V. Bradt, R. E. Rothschild, and J.H. Swank, Astron. Astrophys. Suppl. Ser. 97, 355 (1993).

[38] F. Marshall and J. H. Swank, GRB Circular Network 1996 (2003); also see http://gcn.gsfc.nasa.gov/gcn3/1996.gcn3

[39] E. Berger, A. M. Soderberg, and D. A. Frail, GCN Notice No. 2014, 2003; also see http://www.nrao.edu/pr/2003/ grb030329/

[40] E. Berger et al., Astrophys. J. 599, 408 (2003).

[41] J. Greiner et al., Nature (London) 426, 157 (2003).

[42] K.Z. Stanek et al., American Astronomical Society Meeting 203 (2003); also see http://www.aas.org/ publications/baas/v35n5/aas203/56.htm

[43] P. A. Mazzali et al., Astrophys. J. Lett. 599, L95 (2003).

[44] J. Hjorth et al., Nature (London) 423, 847 (2003).

[45] J. Greiner et al., Nature (London) 426, 157 (2003).

[46] S.E. Woosley, R.G. Eastman, and B.P. Schmidt, Astrophys. J. 516, 788 (1999).

[47] D. Band et al., Astrophys. J. 413, 281 (1993).

[48] A. Zeh et al., GRB Circular Network 2047 (2003).

[49] W. Li et al., GRB Circular Network 2064 (2003); http:// gcn.gsfc.nasa.gov/gcn3/2064.gcn3

[50] A. M. Beloborodov, Astrophys. J. Lett. 539, L25 (2000).

[51] D. A. Frail, E. Waxman, and S. R. Kulkarni, Astrophys. J. 537, 191 (2000).

[52] B. Abbott et al. (LIGO Scientific Collaboration), Nucl. Instrum. Methods Phys. Res., Sect. A 517, 154 (2004).

[53] W. G. Anderson et al., Phys. Rev. D 63, 042003 (2001).

[54] S.D. Mohanty et al., Classical Quantum Gravity 21, S1831 (2004).

[55] R. A. Araya-Góchez, Mon. Not. R. Astron. Soc. 355, 336 (2004).

[56] B. Abbott et al., Phys. Rev. D 69, 102001 (2004).

[57] K.S. Thorne, in 300 Years of Gravitation (Cambridge University Press, Cambridge, England, 1987), edited S. W. Hawking and W. Israel.

[58] C. W. Misner, K. S. Thorne, and J. A. Wheeler, Gravitation (Freeman, San Francisco, 1973).

[59] H. J. Mosquera Cuesta, Phys. Rev. D 65, 064009 (2002).

[60] R. Ruffini et al., Astrophys. J. Lett. 555, L113 (2001).

[61] P. Astone et al., Phys. Rev. D 71, 042001 (2005).

[62] P. Tricarico et al., Phys. Rev. D 63, 082002 (2001).

[63] G. A. Prodi et al., in Proceedings of the Second Edoardo Amaldi Conference on Gravitational Wave Experiments, CERN-Switzerland, 1997, edited by E. Coccia, G. 
Veneziano, and G. Pizzella (World Scientific, Singapore, 1998), pp. 148-158.

[64] L. S. Finn, S. D. Mohanty, and J. Romano, Phys. Rev. D 60, 121101 (1999).

[65] P. Tricarico, A. Ortolan, and P. Fortini, Classical Quantum Gravity 20, 3523 (2003).

[66] G. Modestino and A. Moleti, Phys. Rev. D 65, 022005 (2002).

[67] P. Astone et al., Astron. Astrophys. Suppl. Ser. 138, 603 (1999).

[68] P. Astone et al., Phys. Rev. D 66, 102002 (2002).
[69] P. Astone et al., Phys. Rev. D 47, 362 (1993).

[70] P. Astone et al., Astropart. Phys. 7, 231 (1997).

[71] L. Amati et al., Astron. Astrophys. Suppl. Ser. 138, 605 (1999).

[72] S. Woosley, Nature (London) 430, 623 (2004), news and views.

[73] S. Y. Sazonov, A. A. Lutovinov, and R. A. Sunyaev, Nature (London) 430, 646 (2004), letters to nature.

[74] A. M. Soderberg et al., Nature (London) 430, 648 (2004), letters to nature.

[75] http://space.mit.edu/HETE/Bursts/Data 